\title{
Staphylococcus aureus small colony variants impair host immunity by activating host cell glycolysis and inducing necroptosis
}

\author{
Tania Wong Fok Lung $\mathbb{1}^{1}$, lan R. Monk $\mathbb{D}^{2}$, Karen P. Acker $\mathbb{1}^{1}$, Andre Mu $\mathbb{1}^{2,3}$, Nancy Wang $\mathbb{(}^{2}$, \\ Sebastián A. Riquelme1, Silvia Pires ${ }^{1}{ }^{1}$, Loreani P. Noguera ${ }^{1}{ }^{1}$, Felix Dach'1, Stanislaw J. Gabryszewski ${ }^{\text {, }}$ \\ Benjamin P. Howden $\mathbb{1 D}^{2,3,4}$ and Alice Prince ${ }^{1 \star}{ }^{1 \star}$
}

\begin{abstract}
Staphylococcus aureus small colony variants (SCVs) are frequently associated with chronic infection, yet they lack expression of many virulence determinants associated with the pathogenicity of wild-type strains. We found that both wild-type $S$. aureus and a $\triangle$ hemB SCV prototype potently activate glycolysis in host cells. Glycolysis and the generation of mitochondrial reactive oxygen species were sufficient to induce necroptosis, a caspase-independent mechanism of host cell death that failed to eradicate S. aureus and instead promoted $\Delta$ hemB SCV pathogenicity. To support ongoing glycolytic activity, the $\Delta$ hemB SCV induced over a 100-fold increase in the expression of fumC, which encodes an enzyme that catalyses the degradatin of fumarate, an inhibitor of glycolysis. Consistent with fumC-dependent depletion of local fumarate, the $\triangle$ hemB SCV failed to elicit trained immunity and protection from a secondary infectious challenge in the skin. The reliance of the $S$. aureus SCV population on glycolysis accounts for much of its role in the pathogenesis of $S$. aureus skin infection.
\end{abstract}

T he bacterium Staphylococcus aureus is a versatile human pathogen associated with diverse clinical syndromes, ranging from fulminant sepsis to more persistent subacute infection, as often occurs in endocarditis, chronic pneumonia, osteomyelitis and in the skin and soft tissues. While toxins have been shown to be critical in the initial stages of pathogenesis ${ }^{2-5}$, the S. aureus properties responsible for such indolent infections are not as well established. SCVs of $S$. aureus have long been associated with chronic infection ${ }^{6}$. SCVs often harbour inactivating mutations in genes associated with the production of the terminal electron transport chain components haeme and menaquinone ${ }^{7}$. As a result, they share major metabolic features including decreased utilization of the tricarboxylic acid (TCA) cycle and oxidative phosphorylation and upregulation of glycolysis to generate $\mathrm{ATP}^{8}$. They are often overlooked in clinical microbiology laboratories due to their slow growth and instability in vitro 9 . While their ability to cause persistent infection has been attributed to antimicrobial resistance, intracellular survival and limited production of toxins necessary to elicit immune clearance ${ }^{10}$, exactly how SCV s are selected in the setting of chronic infection is still unclear.

There are important immunological consequences associated with microbial induction of host metabolic activity, particularly glycolysis $^{11,12}$. Keratinocytes respond to wild type (WT) S. aureus by increasing glycolytic activity and generating HIF $1 \alpha$ and IL- $1 \beta^{13}$. We postulated that SCVs activate a metabolic response in host cells, distinctive from that activated in response to more virulent $S$. aureus strains, which enables them to persist. In this study, we demonstrate that by potently activating host glycolysis and mitochondrial reactive oxygen species (ROS), WT S. aureus and a $\Delta h e m B$ SCV prototype stimulate necroptosis in keratinocytes, a form of cell death that eliminates the host cell but releases viable staphylococci ${ }^{14}$ and promotes SCV persistence. Moreover, the $\triangle$ hemB SCV upregulates bacterial fum $C$ expression, promoting the degradation of local fumarate and inhibiting the generation of trained immunity that confers protection against secondary staphylococcal challenge.

\section{Results}

Construction and characterization of a $\Delta$ hemB mutant in USA300 LAC. The phenotypic and metabolic consequences of a hemB deletion in the $S$. aureus 8325-4 background as well as mutations in hemB, menD and thy $A$ in numerous clinical isolates have previously been characterized ${ }^{8}$. We used the $\Delta$ hemB $8325-4$ mutant as a historical SCV control ${ }^{15}$ and constructed a hemB deletion mutant and its complement by gene restoration on the chromosome in the clinically relevant USA300 LAC background (Extended Data Fig. 1a), which were confirmed by whole genome sequencing (Supplementary Table 1). The use of two SCV prototypes is of importance as the historical SCV was constructed in a strain that has undergone extensive ultraviolet mutagenesis ${ }^{16}$ and has defects in the activation of the stress response regulator $\operatorname{SigB}^{17,18}$ that could impact metabolism. The $\Delta$ hemB LAC mutant formed pinpoint colonies on BHI agar but exhibited WT-sized, haemolytic colonies on blood agar, presumably by scavenging haemin from the media, consistent with the loss of $\delta$-aminolevulinic acid dehydratase ${ }^{19}$ (Fig. 1a and Extended Data Fig. 1b). The expression of the global regulator $a g r^{20}$ from the $\Delta h e m B$ LAC mutant was decreased whereas those of the genes $h l a, l u k A, l u k E$, lukS and $h l g C$ encoding $\alpha$-haemolysin (Hla) and subunits of bi-component toxins were all increased, as was expression of $f n b A$, which encodes the fibronectin-binding protein (Fig. 1b). This is in contrast to the historical $\Delta h e m B$ mutant

'Department of Pediatrics, Vagelos College of Physicians \& Surgeons, Columbia University, New York, NY, USA. ${ }^{2}$ Department of Microbiology and Immunology, University of Melbourne at the Peter Doherty Institute for Infection and Immunity, Melbourne, Victoria, Australia. ${ }^{3}$ Microbiological Diagnostic Unit Public Health Laboratory, University of Melbourne at the Peter Doherty Institute for Infection and Immunity, Melbourne, Victoria, Australia. ${ }^{4}$ Department of Infectious Diseases, Austin Health, Heidelberg, Victoria, Australia. *e-mail: asp7@cumc.columbia.edu 
that has reduced hla expression ${ }^{21}$. However, upregulated expression of toxin-encoding genes in the $\Delta$ hemB LAC mutant did not result in increased protein levels (Fig. 1c). WT LAC and the $\Delta$ hemB LAC mutant produced similar levels of Hla in the presence of haemin (Fig. 1c), consistent with the $\beta$-haemolysis observed on blood agar (Fig. 1a). The WT and $\Delta$ hemB mutant strains also produced similar levels of LukA during exponential growth phase and the $\Delta h e m B$ mutant made more LukA/D/E than WT LAC in the presence of haemin (Fig. 1c), indicating that this SCV is probably capable of toxin expression in vivo. The complemented mutant strain displayed a WT phenotype (Fig. 1a-c).

The $\Delta$ hemB SCV induces a distinct metabolic response as compared with WT $S$. aureus. The major property differentiating the SCVs as a group and WT S. aureus strains is their altered metabolic activity ${ }^{8}$. Just as bacteria adapt their metabolic activity in specific settings, host cells similarly respond to bacterial infection with metabolic changes ${ }^{13,22}$. We predicted that the $\Delta$ hemB mutant would induce a distinct metabolic response in comparison to the parent strain. A global metabolome analysis of the supernatants of infected human peripheral blood mononuclear cells (PBMCs) illustrated the different metabolic responses induced by the $\Delta$ hemB LAC mutant as compared with the WT or complemented strains (Fig. 1d). This is shown by the distinct clustering of all metabolites across the different biological groups by partial least-squares analysis (Fig. 1d). Major differences in the levels of extracellular $\mathrm{L}$-arginine, $\mathrm{D}$-proline, succinate, $\mathrm{L}$-ornithine, adenine and uridine5-monophosphate were observed during infection with the SCVs versus the WT strain (Fig. 1e).

An analysis of the lower-abundance metabolites revealed less fumaric acid, $\mathrm{N}$-acetyl-glutamine, $\mathrm{N}$-acetyl-DL-methionine and $N$-acetyl-L-phenylalanine in the extracellular metabolome of $\Delta$ hemB SCV-infected PBMCs (Fig. 1f), consistent with the production of these metabolites during infection with the WT strain. Focusing specifically on the abundance of fumarate, we observed a decrease associated with the PBMCs infected with the $\Delta$ hemB SCV as compared with either the WT or complemented control (Fig. 1g). The distinctive metabolome profile including lower levels of fumarate associated with $\Delta$ hemB infection was corroborated in a similar analysis of infected human monocytes (THP-1 cells) (Extended Data Fig. 1c-f) and reflects the combined products of both host and pathogen metabolism. While all of the $S$. aureus strains tested induced host cell glycolysis, the $\Delta$ hemB SCVs stimulated significantly more glycolytic activity in THP-1 cells than the WT strain (Fig. 1h). This was indicated by a higher extracellular acidification rate (ECAR) in Seahorse extracellular flux assays. The overall contribution of bacterial metabolism to the changes in ECAR was negligible (Fig. 1h), emphasizing the influence of the bacteria on the infected host cells. These S. aureus strains also stimulated keratinocyte glycolysis (Fig. 1i).

S. aureus stimulates necroptosis by inducing host glycolysis. Induction of glycolysis has been linked to the activation of necroptosis $^{23,24}$. This cell death pathway is mediated by the RIP kinases that induce the phosphorylation of MLKL, resulting in oligomerization and pore formation ${ }^{25,26}$. S. aureus can induce necroptotic cell death, which has been shown to be toxin-dependent ${ }^{27,28}$. Necroptosis, in contrast to the caspase-1-associated mechanisms of cell death, does not kill $S$. aureus but releases viable organisms ${ }^{14}$. We observed that WT $S$. aureus and the $\Delta$ hemB SCV induced necroptosis in human primary keratinocytes, indicated by the phosphorylation of MLKL (PMLKL), a response that was inhibited in the presence of a glycolytic inhibitor, 2-deoxyglucose (2-DG; Fig. 2a and Extended Data Fig. 2a). Bacterial glycolytic activity was required for the induction of host glycolysis ${ }^{13}$ and necroptosis as neither heat-killed bacteria nor a $\Delta p y k$ mutant (deficient in glycolysis) ${ }^{13,29}$ that cannot induce host glycolysis ${ }^{13}$ stimulated PMLKL whereas a $\Delta p c k A$ mutant (deficient in gluconeogenesis ${ }^{13,29}$ ) was fully capable of inducing necroptosis (Fig. 2b).

It seemed likely that glycolysis contributes to the induction of necroptosis by fuelling the TCA cycle and generating ROS. The addition of butylated hydroxyanisole (BHA), a ROS scavenger, and an inhibitor of the pyruvate dehydrogenase complex (CPI-613) that blocks aerobic respiration and mitochondrial ROS production ${ }^{30}$ suppressed the induction of PMLKL (Fig. 2a,c). As the inhibitors of glycolysis and ROS could affect both the host and staphylococci, the effects of WT and the $\Delta h e m B$ SCV on host mitochondrial activity were determined by measuring mitochondrial membrane potential, which remained low as ROS production in the infected cells was induced, a response similar to that induced by the positive oxidant control, namely tert-butyl hydroperoxide (TBHP) $)^{31}$ (Fig. 2d,e).

To confirm that the metabolic impact of the $\Delta h e m B$ SCV was sufficient to induce necroptosis, we documented that several toxin-deficient mutants including $\Delta a g r, \Delta h l a$ and $\Delta l u k A B / E D / S F /$ $\triangle h l g A B C$ activated PMLKL in human keratinocytes (Fig. 2f). Moreover, inhibition of the endocytic machinery with cytochalasin $\mathrm{D}$ had no effect on necroptosis (Fig. 2f). All of the toxin-deficient mutants stimulated glycolysis in keratinocytes (Fig. 2g). Thus, there appears to be a major impact of $S$. aureus metabolic activity, independent of toxin expression, on the bioenergetics of the host cell, sufficient to activate glycolysis, ROS and necroptosis.

S. aureus-induced necroptosis is not dependent on specific ligand-receptor interactions or autophagy. Many studies indicate that necroptosis is initiated by tumour necrosis factor receptor 1 (TNFR1) and toll-like receptor (TLR) stimulation, as well as

Fig. 1 The $S$. aureus SCV prototype $\Delta$ hemB stimulates a distinct metabolic response in host cells. a, Growth of WT LAC, the $\Delta$ hemB LAC mutant and the complemented strain, $\Delta$ hemB (hemB), on BHI agar and sheep blood agar. Different dilutions of bacterial cultures were plated on each agar plate; the image shown is from one representative experiment; $n=3$ independent experiments. b, Expression of $a g r$, fnbA, hla, lukA, lukE, lukS and hlgC by qRT-PCR The data represent the mean \pm s.e.m.; $n=2$ biologically independent experiments in triplicate; $R Q$, relative quantification to WT LAC. $\mathbf{c}$, representative immunoblot showing the presence or absence of the S. aureus toxins Hla, LukA, LukD and LukE from the supernatants of bacterial cultures grown with or without haemin; $n=3$ independent experiments. d, Representative principal component analysis score plots showing principal component 1 ( $P C 1 /$ variate 1) versus PC2/variate 2 of the total extracellular PBMC metabolome; $n=1$ biological sample in triplicate. e, $A$ heatmap of the total extracellular PBMC metabolome; $n=1$ biological sample in triplicate. From top to bottom, the arrows indicate metabolites L-arginine, $D$-proline, succinate, L-ornithine, adenine and uridine-5-monophosphate respectively. $\mathbf{f}, \mathrm{A}$ heatmap of lower-abundance metabolites in the extracellular PBMC metabolome; $n=1$ in triplicate. $\mathbf{g}$, Relative quantification of fumarate in the extracellular metabolome of uninfected and infected PBMCs. The data represent the median with the $95 \%$ confidence interval; $n=1$ biological sample in triplicate. $\mathbf{h}, \mathbf{i}$, The ECAR of uninfected (PBS) host cells (human monocytes, THP-1 cells (h), and human keratinocytes, HEKn cells (i)), and those infected with WT LAC, $\Delta$ hemB LAC and the complemented strain or WT 8325-4 and $\Delta$ hemB $8325-4$ following sequential addition of glucose to stimulate glycolysis, oligomycin to inhibit mitochondrial respiration and shift energy production towards glycolysis, and 2-DG to inhibit glycolysis. The ECAR of staphylococci at the same concentration was also measured in the absence of host cells. Each data point is the mean \pm s.e.m.; $n=3$ biologically independent experiments. The single asterisks denote statistical differences with respect to the uninfected cells; the double asterisks denote differences between the $\Delta$ hemB-infected cells and those infected with the WT or complemented strains; $P<0.001$ by two-way ANOVA. 
autophagy, since the autophagy machinery serves as a platform for the assembly of the necrosome complex ${ }^{32,33}$. However, these alternative pathways were excluded. The $\Delta$ hemB SCV induced at least as much LC3B production, an autophagy marker, as did the WT strain (Fig. 3a). Inhibition of the early stages of autophagy by wortmannin (WM) or 3-methyladenine (3-MA) or the late stages with a

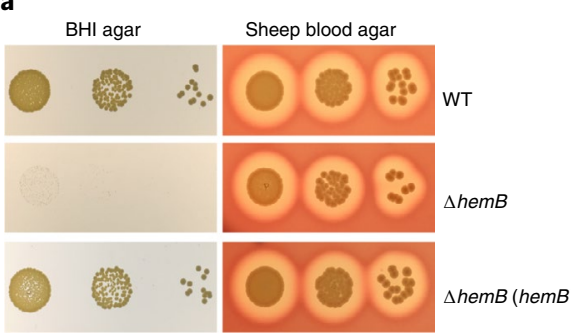

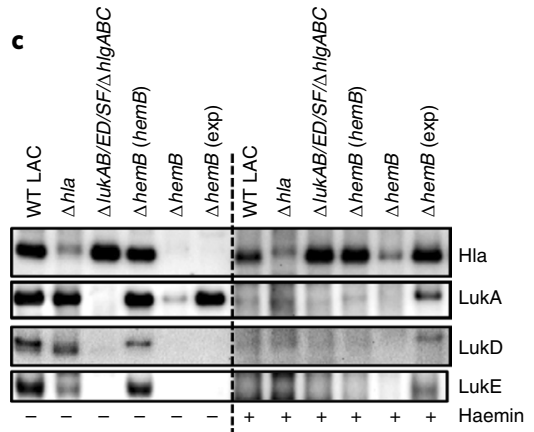

d

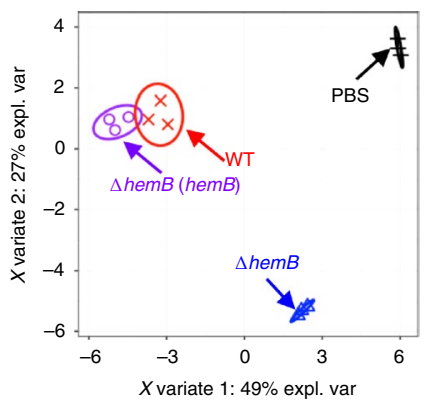

g

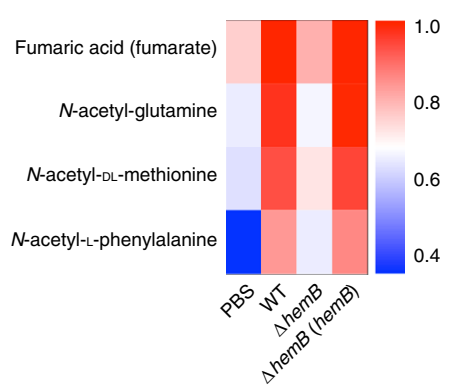

h

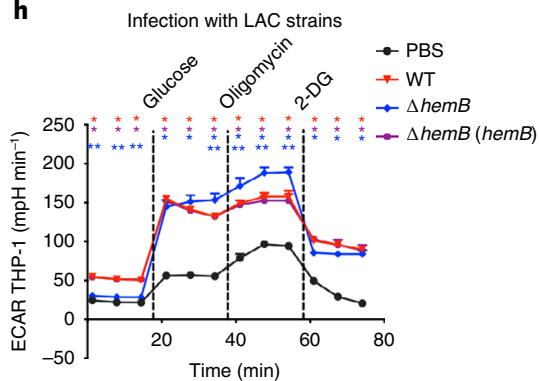

i

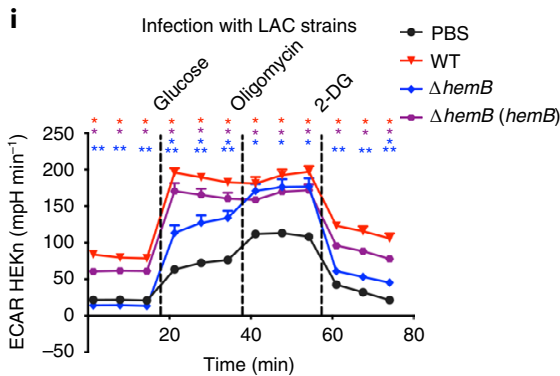

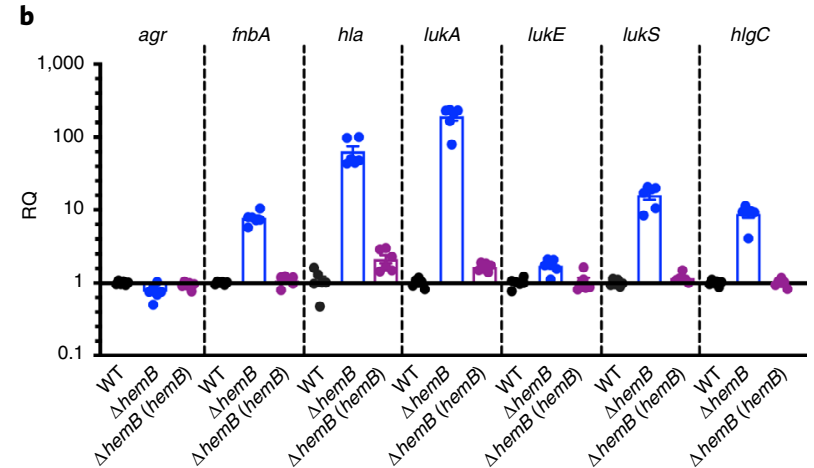

e

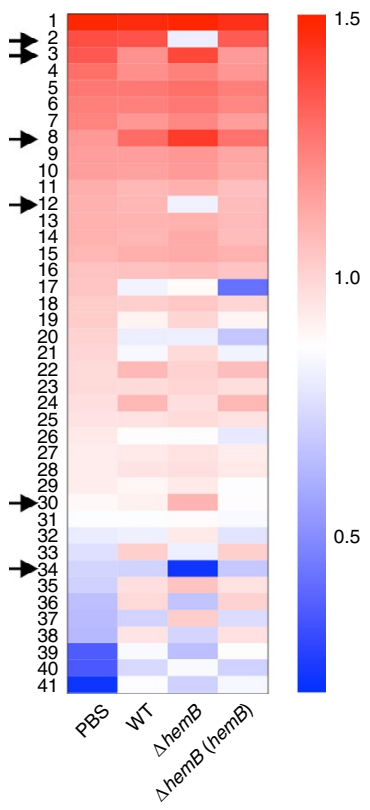

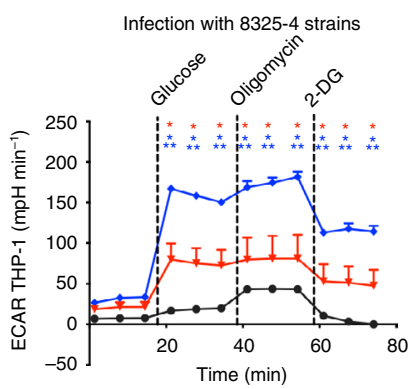
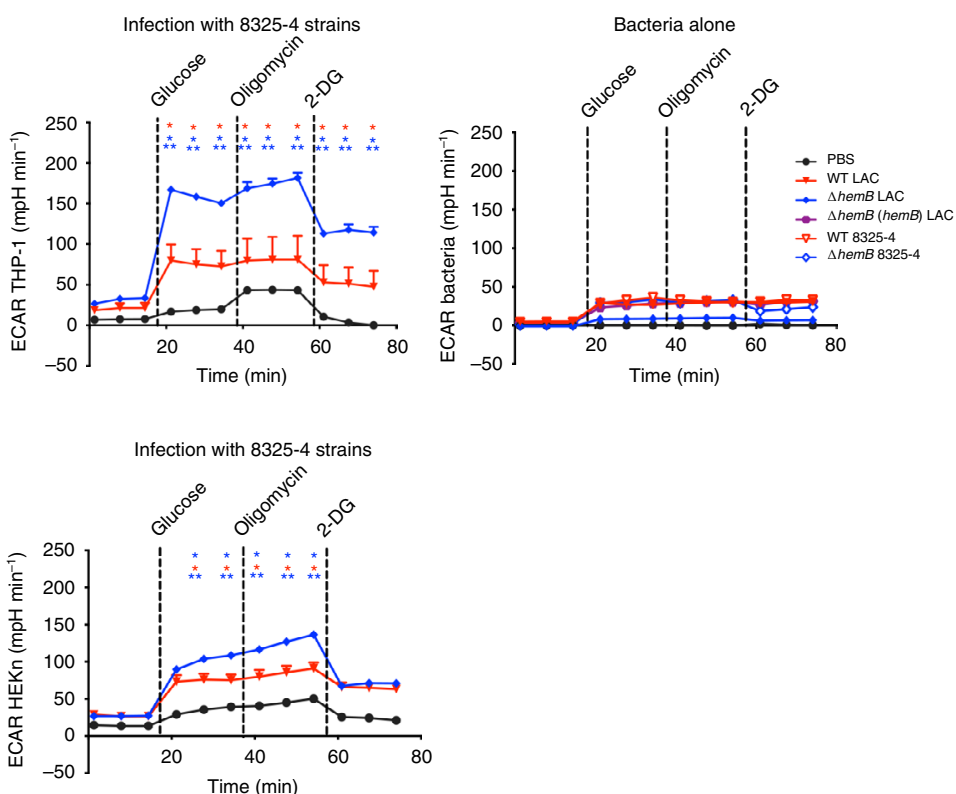


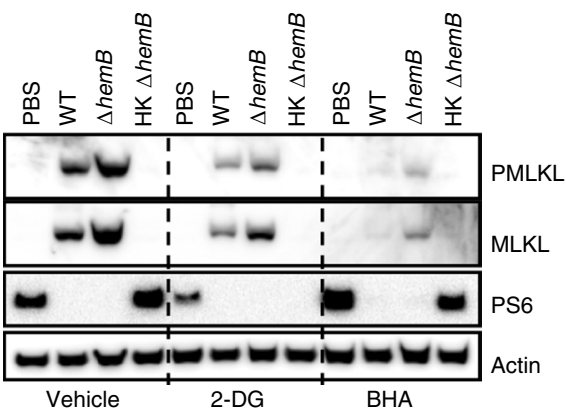

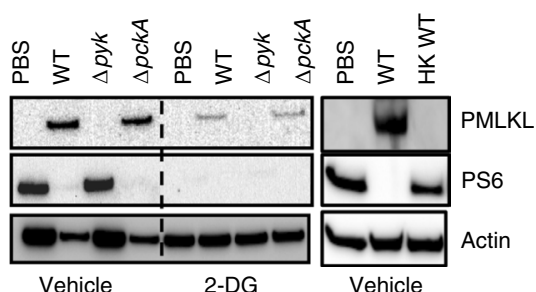

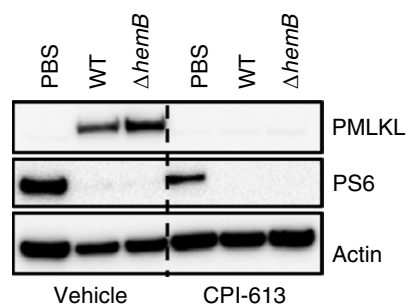

d
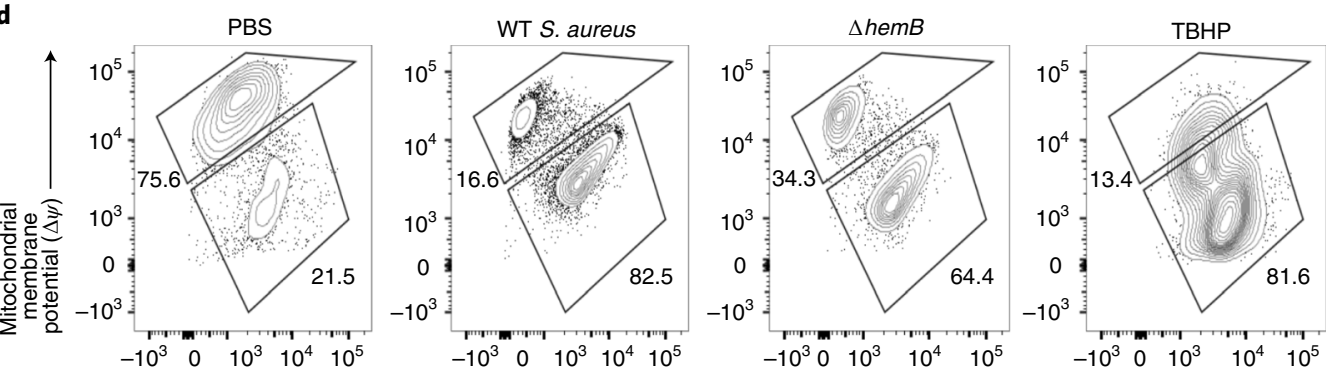

e
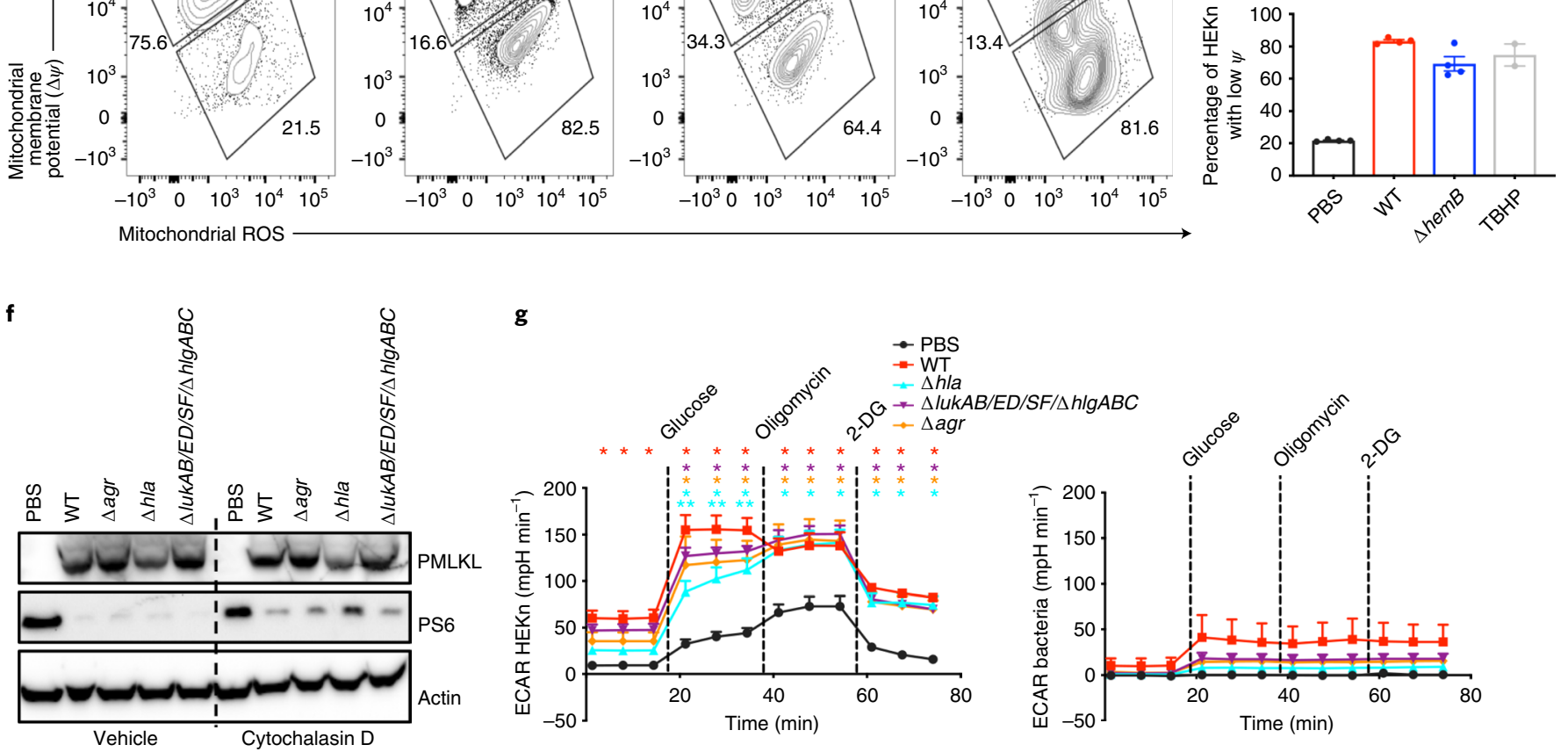

Fig. 2 | S. aureus stimulates necroptosis via the induction of host glycolysis. a-c, Representative immunoblots showing the induction of PMLKL, a marker for necroptosis, and PS6, a marker for proliferative activity, in HEKn cells exposed to PBS, WT S. aureus 8325-4, $\triangle$ hemB 8325-4 or heat-killed (HK) $\Delta$ hemB 8325-4 for $4 \mathrm{~h}$ in the presence and absence of a glycolytic inhibitor, 2-DG, or a ROS scavenger, BHA (a), or to WT LAC, HK WT LAC, $\triangle$ pyk LAC or $\triangle p c k A$ LAC for $24 \mathrm{~h}$ in the presence and absence of 2-DG (b) or to WT 8325-4 or $\Delta$ hemB 8325-4 for $4 \mathrm{~h}$ in the presence and absence of an inhibitor of the pyruvate dehydrogenase complex, $\mathrm{CPI}-613$ (c). For each immunoblot, $n=3$ independent experiments. $\mathbf{d}, \mathrm{A}$ density plot from a representative experiment showing mitochondrial ROS production and membrane potential in HEKn cells exposed to PBS, WT 8325-4, $\Delta$ hemB 8325-4 or an inducer of mitochondrial ROS, THBP, for $3 h ; n=2$ independent experiments in triplicate. e, Mitochondrial ROS production and membrane potential from d. The data represent the mean \pm s.e.m. $n=2$ independent experiments. $\mathbf{f}$, A representative immunoblot showing the induction of PMLKL and PS6 in HEKn cells exposed to PBS, WT LAC, $\Delta$ agr LAC, $\Delta$ hla LAC or $\triangle / u k A B / E D / S F / \triangle h l g A B C L A C$ for $4 \mathrm{~h}$ with or without cytochalasin $D ; n=2$ independent experiments. $g$, The ECAR of uninfected (PBS) HEKn cells and those infected with WT LAC, $\triangle$ agr LAC, $\Delta$ hla LAC or $\Delta / u k A B / E D / S F / \triangle h l g A B C$ LAC. Each data point is the mean \pm s.e.m.; $n=3$ independent experiments. The single asterisks denote statistical differences with respect to the uninfected cells; the double asterisks show statistical differences between the $\Delta$ hla-infected cells and those infected with the other strains; $P<0.001$ by two-way ANOVA. The glycolytic activity of the bacteria alone is shown.

chloroquine (CQ) did not impact the stimulation of PMLKL during infection of keratinocytes (Fig. 3a). The TNFR1 and TLR2 pathways were also excluded. Short interfering RNA to knockdown Tnfrl or Tlr2 in keratinocytes did not prevent the induction of necroptosis (Fig. 3b,c), and TLR2 agonists such as heat-killed S. aureus, lipoarabinomannan (LAM) or Pam3Cys did not activate PMLKL (Fig. 3d).

$\Delta$ hemB SCV-induced necroptosis interferes with bacterial clearance. Given that both WT $S$. aureus and the $\Delta$ hemB SCV induce necroptosis, we next examined how this impacts pathogenesis using a murine model of skin infection. We hypothesized that induction of necroptosis by the $\Delta$ hemB SCV would interfere with $S$. aureus clearance from the skin, as this form of host cell death does not kill staphylococci ${ }^{14}$. We compared intracellular survival of WT LAC, the $\Delta$ hemB LAC mutant and the complemented strain in bone marrow-derived macrophages (BMDMs) from WT and $M l k l^{-1-}$ mice. Similar numbers of colony-forming units (CFUs) were obtained at $1 \mathrm{~h}$ post-infection, indicating equivalent bacterial uptake (Fig. $4 \mathrm{a}$ ). All three bacterial strains were cleared in the presence or absence of necroptosis at 4 and $24 \mathrm{~h}$ post-infection (Fig. $4 \mathrm{a}$ ), confirming that necroptosis does not kill staphylococci. Of note, fewer $\Delta$ hemB LAC CFUs were observed in $M l k l^{-1-}$ BMDMs compared to WT BMDMs 
a

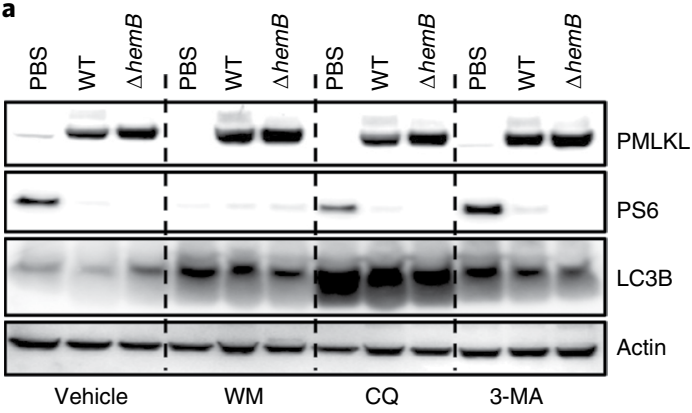

C

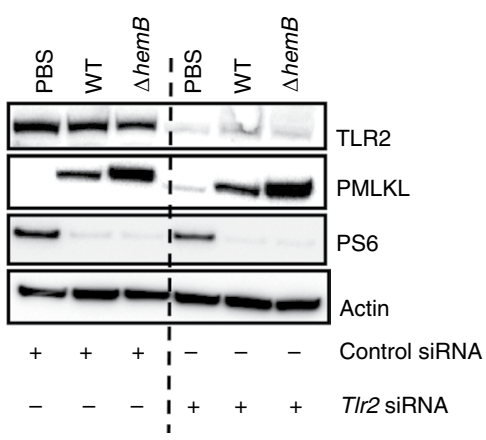

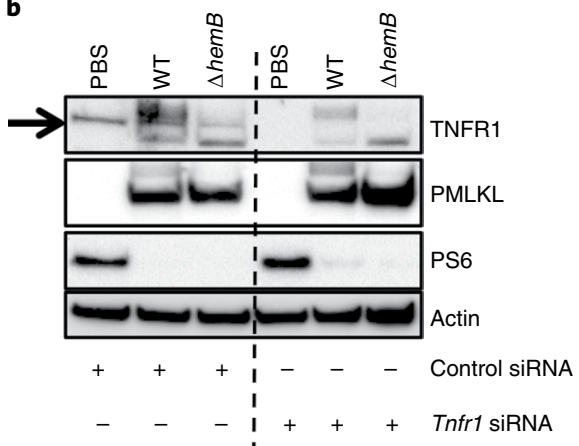

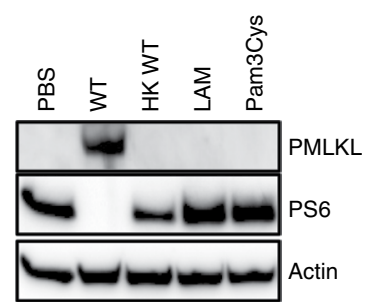

Fig. 3 | S. aureus-induced necroptosis is independent of autophagy or ligand-receptor interactions. a-d, Representative immunoblots showing the induction of PMLKL and PS6 in HEKn cells exposed to PBS, WT 8325-4 or $\Delta$ hemB 8325-4 and treated with the autophagy inhibitors WM, CQ and 3-MA (a), control siRNA or Tnfr1 siRNA (b), control siRNA or TIr2 siRNA (c) and the TLR2 agonists LAM and Pam3Cys (d). The arrow in $\mathbf{b}$ indicates TNFR1. For each immunoblot, $n=3$ independent experiments.

$24 \mathrm{~h}$ post-infection (Fig. $4 \mathrm{~b}$ ), suggesting that necroptosis promotes $\Delta$ hemB persistence.

As has been noted by others ${ }^{34,35}$, primary infection with the $\Delta$ hemB SCVs was attenuated in vivo as demonstrated by smaller skin lesions (dermonecrosis) and lower bacterial burden in a murine model of cutaneous infection (Fig. 4c,d). In spite of the decreased bacterial load, the $\triangle$ hemB LAC SCV s persisted for at least 5 days (Fig. 4 d) and recruited similar numbers of neutrophils, interstitial macrophages, Ly6C $\mathrm{C}^{-}$monocytes, Ly6C $\mathrm{C}^{+}$monocytes, CD $11 \mathrm{~b}^{+}$ dendritic cells (DCs), Langerhans DCs, Langerhans cells and plasmacytoid DCs (pDCs) (Fig. 4e) but less of the proinflammatory cytokines IL-1 $\beta$, TNF $\alpha$, IL-6 and KC (Fig. 4f) than did WT LAC at the site of infection. Similar results were obtained with the $\Delta$ hemB 8325-4 mutant, which recruited a similar immune response as the WT strain except for significantly fewer Langerhans cells $(P<0.01)$ (Extended Data Fig. 2b,c). However, the levels of induction of proinflammatory cytokines by WT $8325-4$ and the $\Delta$ hemB mutant were not statistically different, except for IL-1 $\beta$ and KC (Extended Data Fig. 2d).

We next investigated how necroptosis contributes to the host response, comparing the clearance of WT LAC and the $\Delta h e m B$ LAC SCV from the skin of $\mathrm{Mlkl}^{-1-}$ mice, unable to initiate necroptosis (Fig. 4d and Extended Data Fig. 2b). No statistically significant differences were observed in bacterial burdens in the WT C57BL/6 and $M l k l^{-1-}$ mice infected with WT LAC (Fig. 4d) or WT 8325-4 (Extended Data Fig. 2b). However, in contrast to the indolent infection that persisted in WT C57BL/6 mice, both $\Delta$ hemB SCVs failed to establish infection in $M l k l^{-1-}$ mice (Fig. $4 \mathrm{~d}$ and Extended Data Fig. 2b), suggesting that the ability of the host to activate necroptosis is required for the persistent infection caused by the $\Delta$ hemB SCVs in the skin. No significant differences in immune cell recruitment or cytokine production were observed during infection of WT C57BL/6 versus $M l \mathrm{kl}^{-/-}$mice at five days post-infection (Fig. 4e,f and Extended Data Fig. 2c,d).
Compared to the parental strain, the $\Delta$ hemB 8325-4 SCV was also more readily cleared from the Ripk1d mice, which cannot initiate necroptosis, and evoked a limited innate immune response and less IL-1 $\beta$, TNF $\alpha$, KC and MIP2 production (Extended Data Fig. $2 \mathrm{~b}-\mathrm{d}$ ). Decreased amounts of proinflammatory cytokines (IL-1 $\beta$, TNF, IL-6 and MIP-2) were induced in the skin of Ripk1d mice compared to WT C57BL/6 mice following infection with the $\Delta h e m B$ 8325-4 mutant (Extended Data Fig. 2d). Overall, data obtained with $\Delta$ hemB mutants in both genetic backgrounds indicate that SCV pathogenicity is dependent on the ability of the organisms to activate necroptosis.

The $\Delta$ hemB SCV fails to induce trained immunity. Although the ability of SCVs to induce necroptosis contributes to pathogenicity, this does not fully explain how the SCVs, which are clearly attenuated in other ways, are so successful in a clinical setting. An important consequence of infection with WT S. aureus is the activation of local memory in the skin, a response that is conferred by macrophages and promotes more effective bacterial clearance following secondary exposure ${ }^{36,37}$. Metabolic changes that result in epigenetic reprogramming and increased transcription of proinflammatory cytokines following secondary exposure are the hallmark of trained immunity in macrophages ${ }^{38}$. Given the distinct metabolic signature observed during $\Delta$ hemB SCV infection (Fig. 1d and Extended Data Fig. 2c), we postulated that the host metabolic response induced by $\Delta$ hemB SCVs might limit their ability to elicit trained immunity. Skin infection in C57BL/6 mice was initiated by either WT LAC or the $\Delta$ hemB LAC SCV. Primary infection with WT S. aureus, which normally resolves within 15-18 days, elicited protective immunity as evidenced by significantly decreased dermonecrosis and CFUs when the same site was reinfected 28 days later (Fig. 5a-c). In contrast, primary infection with the $\Delta$ hemB SCV failed to induce protection against a secondary challenge with WT $S$. aureus; the secondary lesions in these mice were larger and the bacterial burden 
C57BL/6 BMDMs WT

- C57BL/6 BMDMs $\triangle$ hemB $(h e m B)$

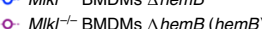

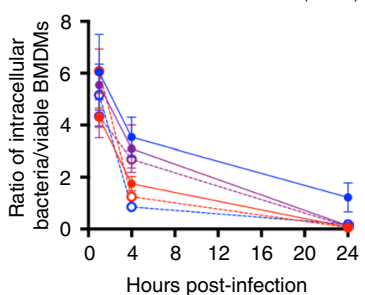

Hours post-infection b

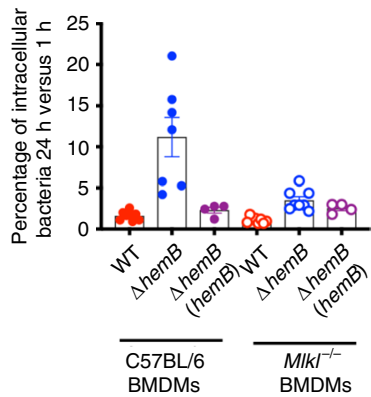

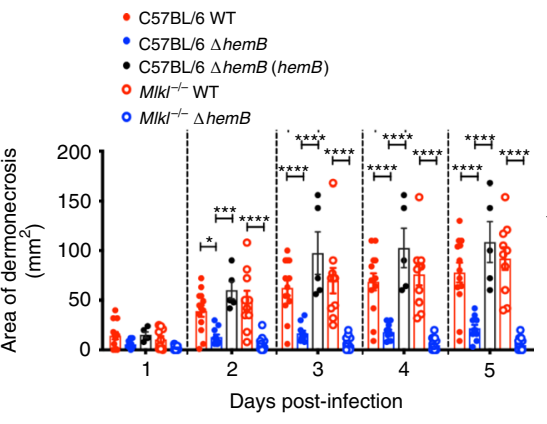

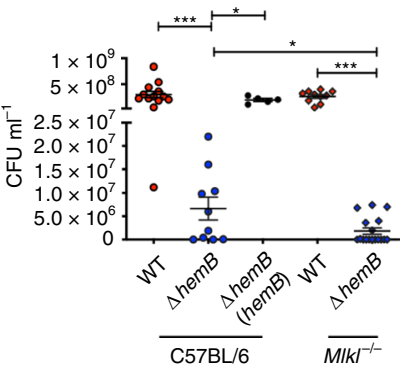

e
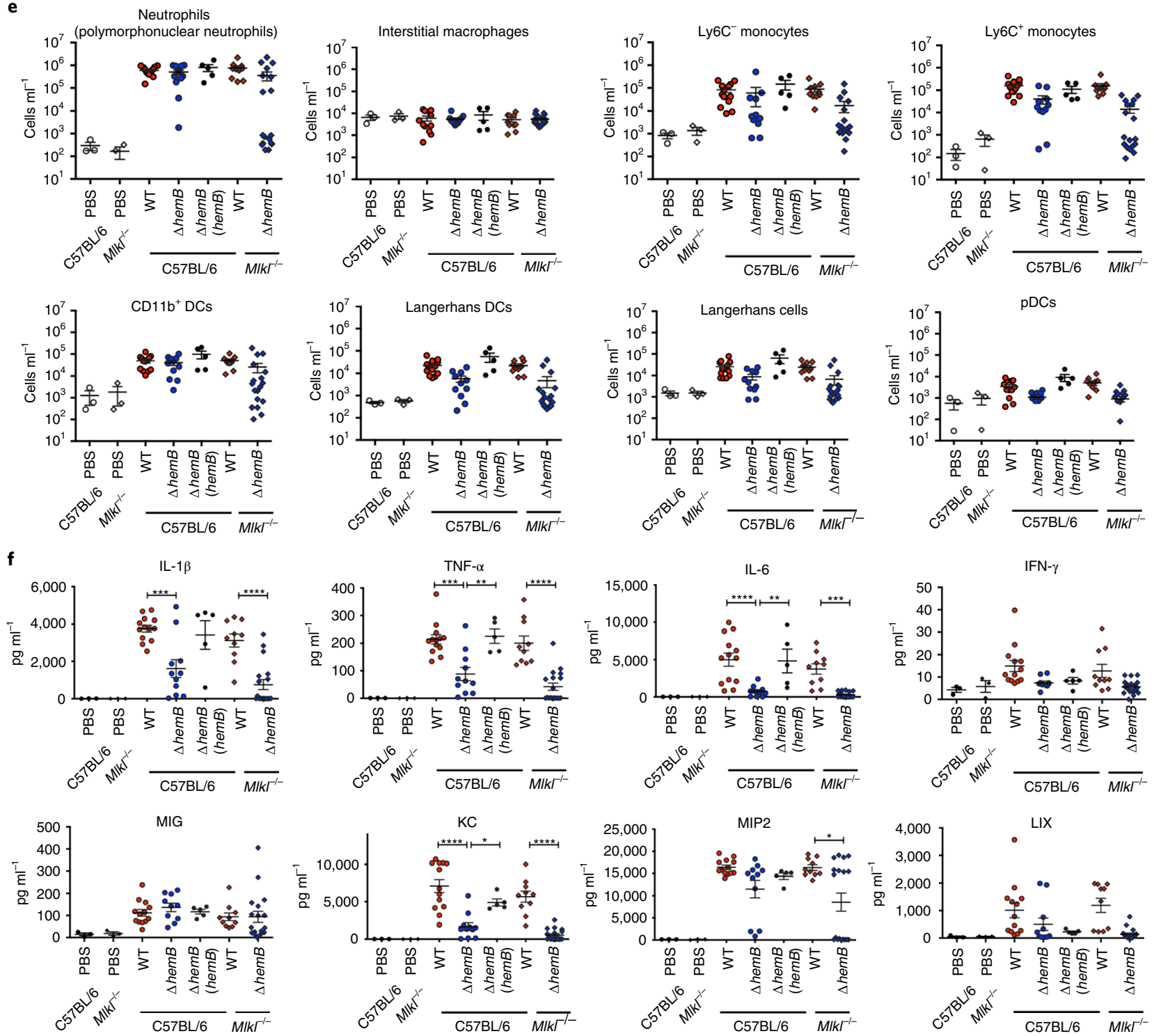

Fig. 4 | $\Delta$ hemB-induced necroptosis impairs bacterial clearance. a, Intracellular survival of WT LAC, the $\triangle$ hemB LAC mutant and the complemented strain, $\Delta$ hemB (hemB), in WT or Mlkl-/- BMDMs at $1 \mathrm{~h}, 4 \mathrm{~h}$ and $24 \mathrm{~h}$ post-infection; $n=2$ biologically independent experiments. b, Intracellular bacterial survival from $\mathbf{a}$ at $24 \mathrm{~h}$ post-infection as a percentage of the intracellular survival at $1 \mathrm{~h}$ post-infection; $n=2$ independent experiments. $\mathbf{c}-\mathbf{f}$, Outcomes of skin infection in WT C57BL/6 and Mlk/-1- mice 5 days post-infection with WT LAC, $\Delta$ hemB LAC or $\Delta$ hemB (hemB) LAC. c, Dermonecrosis from days 1-5 post-infection. d, The bacterial load in WT C57BL/6 and M/k/-/- mice 5 days post-infection. e, Innate immune cell populations recruited at the site of infection at day 5. f, Cytokine measurements from skin biopsies of the infected sites at day 5 . For $\mathbf{a}-\mathbf{f}$, the data represent the mean $\pm \mathrm{s}$.e.m. For $\mathbf{c}-\mathbf{f}, n=13$ (C57BL/6 WT LAC), 10 (C57BL/6 $\Delta$ hemB), 5 (C57BL/6 $\Delta$ hemB (hemB)), 10 (Mlkl-/-WT LAC) or 17 (Mlkl-/- $\Delta$ hemB) independent animals. Statistical analysis was performed by one-way (d-f) and two-way (c) ANOVA; ${ }^{\star} P<0.05,{ }^{\star \star} P<0.01,{ }^{\star \star \star} P<0.001,{ }^{\star \star \star \star} P<0.0001$. 
was higher than those from mice that initially encountered WT $S$. aureus (Fig. 5b,c). The participation of T and B cells in the memory response to $S$. aureus infection was examined. Both WT C57BL/6 and Rag2 knockout (KO) mice exhibited protection against secondary infection with smaller lesions although no significant differences in CFUs were observed (Fig. 5d,e), findings consistent with recent reports $^{36}$. This indicates that secondary protection against $S$. aureus infection is not solely mediated by adaptive immunity and requires trained immunity. Necroptosis was not essential in inducing trained immunity; $\mathrm{Mlkl}^{-1-}$ mice infected initially with WT S. aureus were protected from a secondary challenge with the WT strain (Fig. 5f,g). Accordingly, we questioned whether a specific component of the metabolic activity of the SCVs inhibits trained immunity.

Increased expression of fumC in the $\Delta$ hemB SCV inhibits trained immunity. The production of fumarate is important in stimulating the epigenetic changes associated with trained immunity in monocytes $^{39}$. As a TCA cycle substrate important in oxidative phosphorylation, fumarate production would be decreased in cells whose metabolic activity is skewed to favour glycolysis, as is the case for the SCV-infected cells, particularly since fumarate itself suppresses glycolysis ${ }^{40}$. We predicted that infection with the $\Delta$ hemB SCV would generate less fumarate, as we observed in vitro (Fig. $1 \mathrm{~g}$ and Extended Data Fig. 1f) and hence would limit the epigenetic changes necessary for trained immunity. We established that less fumarate accumulates in the supernatant of $\Delta h e m B$-infected as compared with WT S. aureus-infected keratinocytes (Fig. 6a). This was consistent with the upregulation of fum $C$, which encodes fumarate hydratase, an enzyme that converts fumarate to malate (Fig. 6b), and correlated with significantly decreased local accumulation of fumarate in infected skin (Fig. 6c). To determine whether increased fum C expression in the SCVs would be sufficient to regulate local concentrations of fumarate, we performed a mixed infection in vivo, demonstrating that even with a $1: 1$ ratio $(\mathrm{WT} / \Delta$ hem $\mathrm{B})$ there was still significantly decreased fumarate accumulation in the skin (Fig. 6c). This effect of the $\Delta$ hemB SCV was apparent in vivo, even though the WT strain significantly outcompeted the $\Delta h e m B$ mutant when infected at either a 1:1 or 1:3 ratio (Fig. 6d,e).

To better appreciate the contribution of increased fum $C$ expression in pathogenesis, a fum $C$ deletion mutant in the LAC background was constructed and tested in the murine skin infection model, predicting that it would stimulate trained immunity by allowing the accumulation of local fumarate. Similar levels of fumarate were present in the skin of mice infected with either the $\Delta$ fumC mutant or WT strain (Fig. 6f). Primary infection with the $\Delta$ fumC mutant was equivalent to the WT strain as demonstrated by similar lesion sizes and bacterial burden (Fig. 6g,h). Mice initially infected with either the $\Delta$ fumC mutant or WT/complemented strains developed local protective immunity following secondary challenge with WT $S$. aureus, as demonstrated by smaller lesions and CFUs (Fig. 6i,j). We verified in vitro that the $\triangle$ fumC LAC mutant stimulated less glycolysis in keratinocytes than the WT or complemented strains, consistent with the anti-glycolytic activity of fumarate (Fig. 6k).

A $\Delta$ hemB $\Delta$ fum $C$ double mutant was generated in the LAC background to enable us to address the importance of upregulated fumC expression in the $\Delta$ hemB background. We predicted that in the absence of fumC, the $\Delta$ hemB $\Delta$ fum $C$ double mutant would no longer inhibit trained immunity. The $\Delta$ hemB $\Delta$ fum $C$ mutant formed pinpoint colonies on BHI agar but WT-sized haemolytic colonies on blood agar, as did the $\Delta$ hemB mutant (Extended Data Fig. 3a). Both the $\Delta h e m B$ and $\Delta h e m B \Delta$ fum $C$ mutants displayed slow growth in liquid medium (Extended Data Fig. 3b). Primary infection with both mutants was attenuated as demonstrated by decreased dermonecrosis, bacterial burden and cytokine production compared to primary infection with the WT strain (Fig. 61,m and Extended Data Fig. 3c). Although both $\Delta$ hemB and $\Delta$ hemB $\Delta$ fumC mutants established indolent infections, primary infection with the $\Delta$ hemB $\Delta$ fum $C$ mutant elicited protective immunity as evidenced by significantly decreased dermonecrosis and bacterial burden when the same site was reinfected 28 days later. This protection was similar to that induced by primary infection with either WT $S$. aureus or the $\Delta$ fum $C$ mutant but not the $\Delta$ hemB mutant (Fig. 6n,o).

Additional mechanisms that might contribute to the induction of local memory by WT but not the SCVs were addressed. The production of IL-1 $\beta$ following Aim 2 stimulation, which has been associated with memory and protective epigenetic changes in the skin $^{41}$, was excluded given that both WT C57BL/6 and Ill $r 1^{-/-}$mice lacking IL-1 $\beta$ signalling were protected from secondary challenge (Extended Data Fig. 4a,b). Nor were there differences in dermonecrosis or S. aureus clearance following primary infection of WT or Aim2 ${ }^{-1-}$ mice (Extended Data Fig. 4c,d). S. aureus also failed to activate Aim2 signalling in keratinocytes (Extended Data Fig. 4e,f). Thus, our results indicate that the metabolic activities of $\Delta h e m B$ SCVs are biologically significant in inhibiting local fumarate accumulation and enhancing their ability to cause chronic infection.

\section{Discussion}

S. aureus SCVs, as modelled by $\Delta$ hemB mutants, are an important component of the staphylococcal communities associated with chronic human infection. Our studies indicate that SCV properties unrelated to their more widely recognized antimicrobial resistance and intracellular survival play a major role in their pathogenicity. The metabolic response induced by SCVs contributes to their success as a cause of chronic infection. We demonstrate that WT $S$. aureus and the $\Delta$ hemB mutant from both the LAC and 8325-4 back-

Fig. 5 | The $\Delta$ hemB SCV fails to induce trained immunity. a-g, WT C57BL/6, Rag2 KO or Mlkl $l^{-1-}$ mice were treated with PBS or infected with WT LAC or $\triangle$ hemB LAC on day 0 and challenged at the same site on day 28 with WT LAC. a, A schematic diagram of in vivo infection. $\mathbf{b}$, Dermonecrosis over the course of WT C57BL/6 infection. The black asterisks denote statistical differences in lesion size with respect to uninfected mice, the red asterisks show statistical differences between the WT-infected and $\Delta$ hemB-infected mice, the purple asterisks show statistical differences between naive and WT-S. aureus-primed mice post-secondary challenge and the orange asterisks denote statistical differences between naive and $\Delta$ hemB-infected mice; $n=3$ (C57BL/6 PBS PBS), 9 (C57BL/6 PBS WT), 11 (C57BL/6 WT WT) or 16 (C57BL/6 $\Delta$ hemB WT) independent mice. c, The bacterial load from b at five days post-secondary challenge. d, Dermonecrosis in WT C57BL/6 and Rag2 KO mice treated with PBS or infected with WT LAC on day 0 and challenged at the same site on day 28 with WT LAC. The black asterisks denote statistical differences in lesion size with respect to uninfected mice; $n=2$ (C57BL/6 or Rag2 KO PBS PBS), 3 (C57BL/6 PBS WT), 3 (C57BL/6 WT WT), 3 (Rag2 KO PBS WT) or 3 (Rag2 KO WT WT) independent mice. e, The bacterial load from d at five days post-secondary challenge. f, Dermonecrosis in Mlkl ${ }^{-1-}$ mice treated with PBS or infected with WT LAC on day 0 and challenged at the same site on day 28 with WT LAC. The black asterisks denote statistical differences in lesion size with respect to uninfected mice and the purple asterisks show

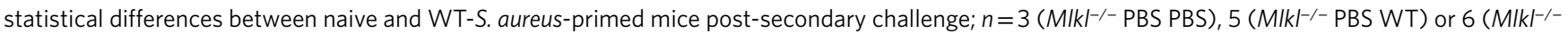
WT WT) independent mice. $\mathbf{g}$, The bacterial burden from $\mathbf{f}$ at five days post-secondary challenge. For $\mathbf{b}-\mathbf{g}$, the data represent the mean \pm s.e.m. Statistical analysis was performed by two-way $(\mathbf{b}, \mathbf{d}, \mathbf{f})$ and one-way $(\mathbf{c}, \mathbf{e}, \mathbf{g})$ ANOVA; ${ }^{\star} P<0.05,{ }^{\star \star} P<0.01,{ }^{\star \star \star} P<0.001,{ }^{\star \star \star \star} P<0.0001 ; P=0.1106(\mathbf{c}), P>0.9999(\mathbf{e})$. NS, not significant. 
grounds induce glycolysis, which in turn activates host cell necroptosis. While the $\Delta$ hemB SCVs are relatively attenuated in their ability to cause local skin infection, their induction of necroptosis does not eliminate staphylococci but instead promotes their persis- tence. Although necroptosis has previously been linked to bacterial toxin production ${ }^{14,27,28}$, we found that the variable expression of toxins by the SCVs was not required to induce necroptosis, which was instead dependent on ROS generated by glycolysis.
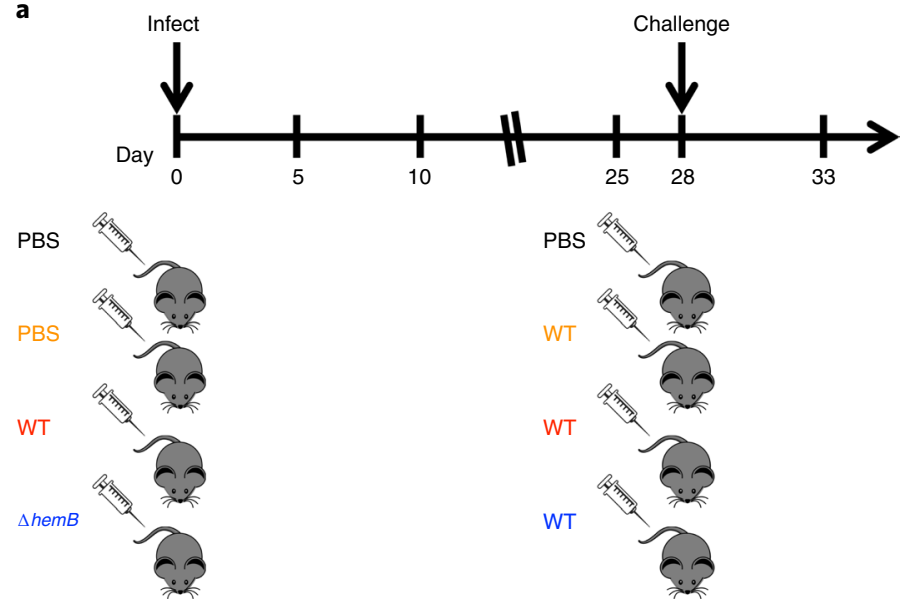

b

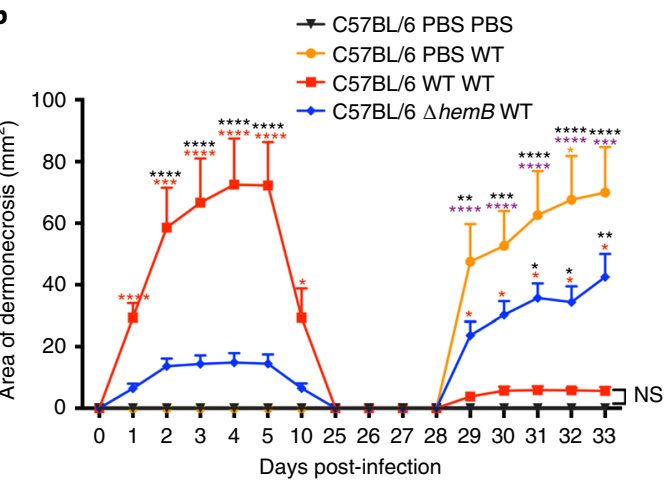

d
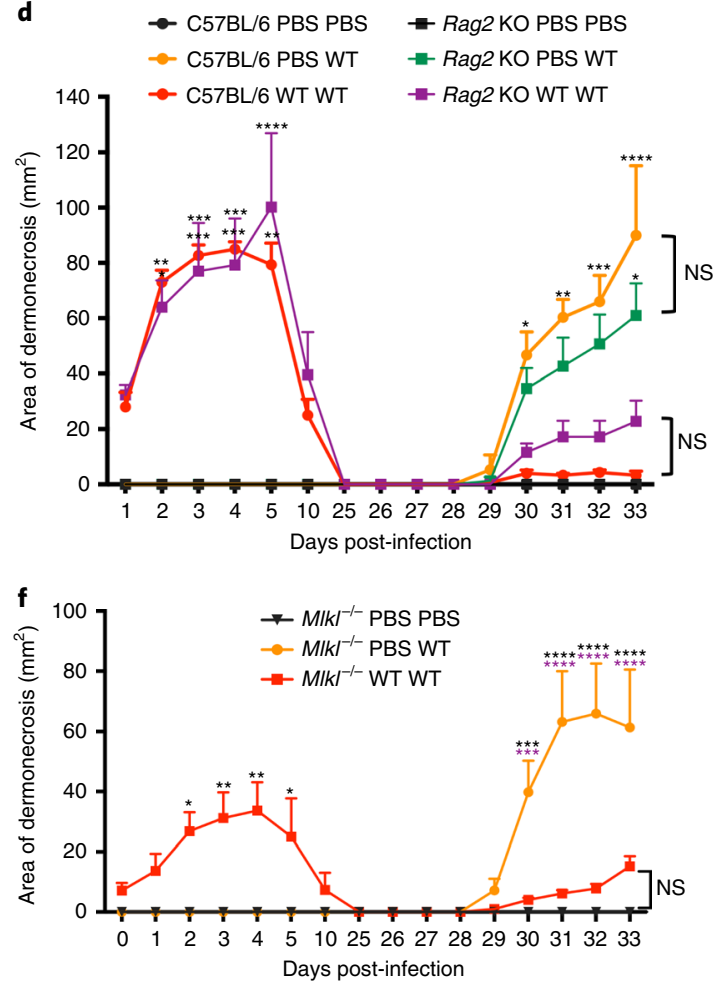

PBS

WT

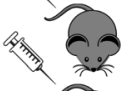

WT

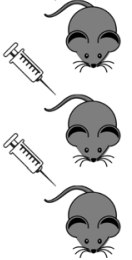

c
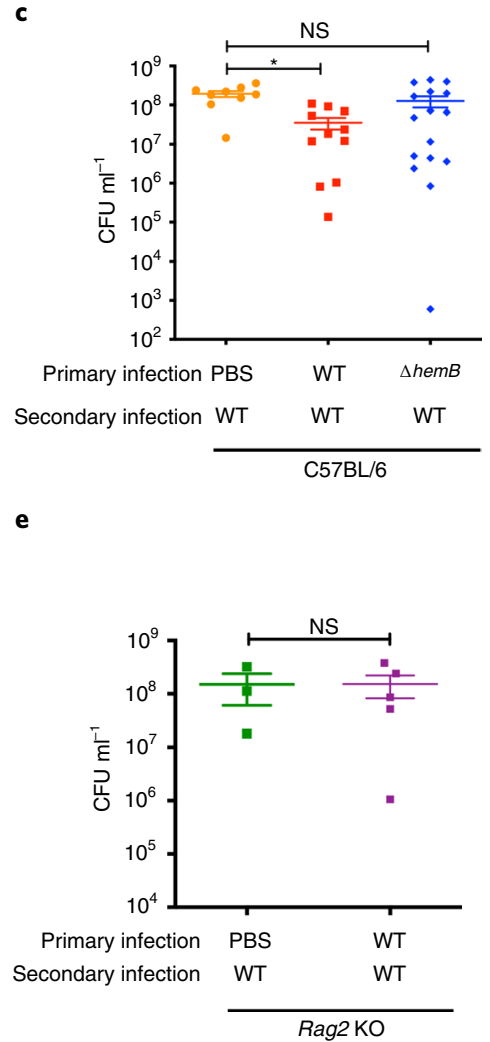

g

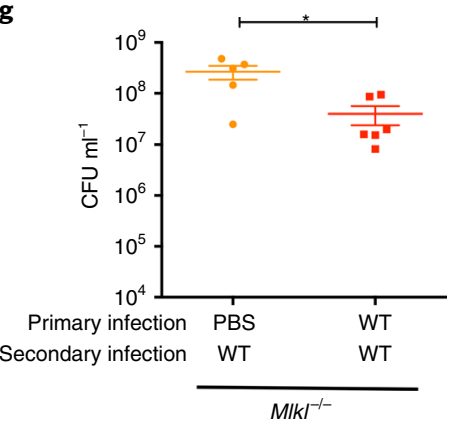


A metabolomic analysis of SCV versus WT S. aureus-infected host cells indicated that a specific metabolomic profile is associated with SCV infection. SCV upregulation of fumC expression depleted local fumarate, both in vitro and in vivo, which correlated with the ability of the $\Delta h e m B$ mutants to evade trained immunity. WT $S$. aureus strains that did not deplete fumarate elicited substantial protection from a secondary infectious challenge. In monocytes, fumarate is essential for the epigenetic changes associated with trained immunity ${ }^{39}$. Since the $\Delta$ hemB SCVs are electron transport chain-deficient and dependent on glycolysis, which is inhibited by fumarate ${ }^{40}$, they must actively regulate the abundance of local fumarate. Even when SCVs comprised only a fraction of the infecting population of staphylococci, as is likely in a clinical infection, their upregulated fumC transcription was sufficient to significantly decrease local levels of fumarate. Our data indicate that SCV evasion of trained immunity in the skin did not correlate with the intensity of the primary immune response, but instead depended on the depletion of local fumarate, adding to the growing body of literature demonstrating the importance of metabolites in innate immune memory ${ }^{11}$.

There are major efforts underway to develop $S$. aureus vaccines based on their expression of toxins and surface adhesins. While these may be highly effective in blocking infection with virulent organisms, the SCVs will remain a challenge. Not only are they protected by slow growth, antimicrobial resistance and their often intracellular niche, but their metabolic activity also predisposes the host to a secondary infection instead of generating protective local immunity.

\section{Methods}

Bacterial culture. The bacterial strains used in this study are listed in Supplementary Table 1. S. aureus USA300 LAC and 8325-4 strains were grown at $37^{\circ} \mathrm{C}$ with shaking in Luria-Bertani broth (LB, BD) and tryptic soy (TS, BD) broth, respectively, or on LB, TS or brain heart infusion (BHI, Sigma) supplemented with $1 \%$ agar (Sigma). The $\Delta$ hemB 8325-4 mutant was cultured in media supplemented with erythromycin $2.5 \mu \mathrm{g} \mathrm{ml}^{-1}$ (Sigma-Aldrich). The $\Delta p y k \mathrm{LAC}$ strain was cultured in media supplemented with sodium pyruvate (Sigma-Aldrich) at a final concentration of $5 \mathrm{mg} \mathrm{ml}^{-1}$. Bacterial inocula were estimated on the basis of the optical density at $600 \mathrm{~nm}\left(\mathrm{OD}_{600 \mathrm{~nm}}\right)$ and verified by retrospective plating on agar plates to determine CFUs. S. aureus recovered from mouse skin biopsies were selected on BBL CHROMagar Staph aureus plates (BD).

Primers and plasmids. The primers and plasmids used in this study are listed in Supplementary Tables 2 and 3.
Construction of $\Delta h e m B$ LAC, $\Delta$ fumC LAC, $\Delta$ hemB $\Delta$ fumC LAC and complemented strains. The hemB and fumC deletion mutants ( $\Delta$ hemB, $\Delta$ fumC) in the LAC background were constructed using the pIMAY-Z allelic exchange plasmid $^{42}$. Upstream and downstream products of hemB and fumC were amplified from LAC genomic DNA with the primer sets $\mathrm{P} 1 / 2, \mathrm{P} 5 / 6$ and $\mathrm{P} 7 / 8, \mathrm{P} 9 / 10$, respectively. The upstream and downstream products of hemB and fum $C$ were joined by splicing by overlap extension PCR with the primers P1/6 and P7/10, respectively, gel extracted and cloned by seamless ligation cloning extract ${ }^{43}$ into Escherichia coli IM08B. The plasmids pIMAY-Z $\Delta$ hemB and pIMAY-Z $\Delta$ fumC were subsequently electroporated into WT LAC and allelic exchange was performed as described by Monk et al. ${ }^{42}$. The LAC $\Delta h e m B$ and $\Delta$ fumC strains were verified by PCR and whole genome sequencing. The $\Delta$ hemB $\Delta$ fumC double mutant was generated by electroporating pIMAY-Z $\Delta$ hemB into the $\triangle f u m C$ strain and verified by PCR and whole genome sequencing. Restoration of the hemB and fum $C$ genes was achieved by cloning the wild-type hemB and fum $C$ genes with the incorporation of a silent restriction enzyme site (SalI or XhoI) by splicing by overlap extension PCR into the gene with the primers P1/3/4/6 and P7/12/11/10, respectively. The procedure was repeated as described above except the pIMAY$\mathrm{ZhemB}^{+}$and pIMAY-Zfum $\mathrm{C}^{+}$constructs were transformed into the LAC $\Delta$ hemB and $\triangle$ fum $C$ strains. This yielded the complemented strains LAC $\Delta$ hemB (hemB) and $\triangle$ fumC $($ fumC)

Isolation of bacterial RNA, complementary DNA synthesis and qRTPCR. Triplicate LB cultures of WT LAC, $\Delta$ hemB and $\Delta$ hemB (hemB) were standardized on the basis of $\mathrm{OD}_{600 \mathrm{~nm}}$. RNAprotect bacteria reagent (QIAGEN) was added to bacterial cultures, vortexed and incubated at room temperature for $10 \mathrm{~min}$. Bacterial cell pellets were collected by centrifugation, frozen and resuspended in $1 \mathrm{ml}$ of TRIsure (Bioline) before lysis by bead beating at 6,000 r.p.m. for $40 \mathrm{~s}$ (zirconia/silica beads (BioSpec), Precellys tissue homogenizer). The debris and beads were pelleted and the supernatants were added to equal volumes of $100 \%$ ethanol. The sample was processed through Direct-zol spin columns (Zymoresearch) with on-column and then off-column DNaseI treatment with Turbo DNaseI (Invitrogen), as described by the manufacturer. The absence of contaminating DNA was verified by PCR with Phire polymerase (Thermo Fisher Scientific) for 35 cycles using gyrB primers (P13/14). RNA was converted to cDNA using Superscript IV reverse transcriptase (Invitrogen). For qRT-PCR, $1 \mu \mathrm{l}$ of cDNA was used with SsoAdvanced Universal SYBR Green Supermix (Bio-Rad) using a MX3000P QPCR system (Agilent Technologies) with the primer sets P15/16, P17/18, $\mathrm{P} 19 / 20, \mathrm{P} 21 / 22, \mathrm{P} 23 / 24, \mathrm{P} 25 / 26, \mathrm{P} 27 / 28$ and $\mathrm{P} 13 / 14$ to amplify agrA, fnbA, hla, lukA, lukE, lukS, hlgC and gyrB, respectively.

For the measurement of fumC expression, overnight cultures of WT LAC and $\Delta$ hemB were subinoculated in TS broth supplemented with $0.5 \%$ glucose and grown statically overnight. The overnight cultures were normalized on the basis of $\mathrm{OD}_{600 \mathrm{~nm}}$ and collected by centrifugation. Bacterial pellets were incubated in lysis buffer ( $50 \mu \mathrm{M}$ Tris-EDTA pH 7.5, $8 \mathrm{U} \mathrm{ml}^{-1}$ mutanolysin (Sigma-Aldrich), $0.018 \mathrm{mg} \mathrm{ml}^{-1}$ lysostaphin (Sigma-Aldrich), $0.05 \mathrm{mg} \mathrm{ml}^{-1}$ lysozyme (SigmaAldrich)) at $37^{\circ} \mathrm{C}$ for $45 \mathrm{~min}$ and TRK lysis buffer (Omega Bio-tek) was added After $10 \mathrm{~min}$ at room temperature, $70 \%$ ethanol was added and samples were transferred to E.Z.N.A RNA isolation columns (Omega Bio-tek). RNA was isolated

Fig. 6 | Overexpression of fumC in $\Delta$ hemB SCVs inhibits trained immunity. a, Fumarate levels in HEKn media; $n=2$ independent experiments. $\mathbf{b}$, Expression of S. aureus fumC in WT and $\Delta$ hemB LAC by qRT-PCR. $n=3$ independent experiments; ${ }^{\star \star} P<0.005$, by Mann-Whitney $U$-test. c, Fumarate in murine skin infected with WT S. aureus ( $n=5$ independent samples), $\Delta$ hemB $(n=7)$ or a mixed inoculum at a $1 / 1$ ratio ( $n=7)$. d, The competitive index of $\Delta$ hemB versus WT S. aureus in skin infected with WT to $\Delta$ hemB ratios of $1 / 1(n=7)$ and $1 / 3(n=3)$. A competitive index of 1 indicates equal colonization capabilities to the parental strain. e, Dermonecrosis in C57BL/6 mice infected with WT S. aureus $(n=5), \Delta$ hemB $(n=7)$ or mixed inocula at ratios of $1 / 1(n=7)$ and $1 / 3(n=3)$. The black asterisks denote statistical differences with respect to uninfected mice; the coloured asterisks denote comparison with WT S. aureus single infection. f, Fumarate in murine skin infected with WT LAC, $\Delta$ fumC LAC or the complemented strain; $n=5$. $\mathbf{g}$, Dermonecrosis at five days post-infection with the strains in $\mathbf{f}$. The black and red asterisks denote statistical differences in lesion size with respect to uninfected mice and between WT and $\Delta$ fumC (fumC)-infected mice, respectively; $n=5$. $\mathbf{h}$, The bacterial load from $\mathbf{g}$ on day 5 . $\mathbf{i}$, Dermonecrosis in WT mice treated with PBS, WT LAC, $\triangle$ fumC LAC or the complemented strain on day 0 and challenged at the same site on day 28 with WT LAC; the black, red and orange asterisks denote statistical differences in lesion size with respect to uninfected mice, WT and $\Delta$ fumC-infected mice, and between naive and primed mice postsecondary challenge, respectively; $n=5$ or 6 . j, The bacterial load from $\mathbf{i}$ at five days post-secondary challenge. $\mathbf{k}$, The ECAR of uninfected (PBS) and WT LAC-, $\triangle$ fumC LAC- or complemented strain-infected HEKn cells. The single asterisks show comparisons to uninfected cells; the double asterisks compare $\Delta$ fumC-infected to WT-infected cells, $n=3$. I, Dermonecrosis at five days post-infection with WT LAC, $\Delta$ hemB or $\Delta$ hemB $\Delta$ fumC LAC. The black asterisks denote statistical differences in lesion size between WT-infected and uninfected mice; the blue and cyan asterisks denote statistical differences in lesion size between WT-infected and $\Delta$ hemB-infected mice, and between WT-infected and $\Delta$ hemB $\Delta$ fumC-infected mice, respectively; $n=4$ or 5 . $\mathbf{m}$, The bacterial load from I on day 5. $\mathbf{n}$, Dermonecrosis in mice treated with PBS, WT LAC, or the $\Delta$ hemB, $\Delta$ hemB $\Delta$ fumC or $\Delta$ fumC LAC mutants on day 0 and challenged at the same site on day 28 with WT LAC. The black, orange and blue asterisks denote statistical differences in lesion size with respect to uninfected mice, between naive or $\Delta$ hemB-primed mice; or between WT LAC and $\Delta$ hemB $\Delta$ fumC or $\Delta$ fumC-primed mice post-secondary challenge; $n=4$ or 5 independent mice per group. o, The bacterial load from $\mathbf{n}$ at day 5. For $\mathbf{a}-\mathbf{0}$, the data represent the mean \pm s.e.m.; ${ }^{\star} P<0.05$, ${ }^{\star \star} P<0.01,{ }^{\star \star \star} P<0.001,{ }^{\star \star \star \star} P<0.0001$ by one-way (c,f,h,j, $\mathbf{m}, \mathbf{0})$ or two-way ANOVA (e,g,i,k,l, n); $P=0.9074,0.8807$ (WT versus $\Delta$ fumC or $\Delta$ fumC (fumC), respectively), 0.9999 ( $\Delta$ fumC versus $\Delta$ fumC (fumC) (f); $P=0.2210$ (o). NS, not significant. 
following the manufacturer's instructions and treated with DNase using the DNAfree DNA removal kit (Invitrogen). Mutltiscribe reverse transcriptase (Applied Biosystems) was used to generate cDNA for qRT-PCRs with Power SYBR Green PCR Mastermix (Applied Biosystems). qRT-PCR was performed using primers for fum $C$ and $16 S$ (P29/30, P31/32) and a StepOne Plus thermal cycler (Applied Biosystems), and data were analysed using the $\Delta \Delta \mathrm{C}_{\mathrm{T}}$ method.

Exoprotein isolation from $S$. aureus. Overnight $S$. aureus cultures were subcultured for $6.5 \mathrm{~h}$ with $(10 \mu \mathrm{M})$ or without haemin (Sigma) and standardized at an $\mathrm{OD}_{600 \mathrm{~nm}}$ of 8 . Bacterial cells were pelleted by centrifugation at 4,000 r.p.m. for $10 \mathrm{~min}$. The supernatants were filtered through a $0.2-\mu \mathrm{m}$-pore-size filter (Nalgene) and precipitated with $10 \%(\mathrm{v} / \mathrm{v})$ trichloroacetic acid (Sigma) at $4{ }^{\circ} \mathrm{C}$ overnight. The precipitated proteins were washed with $100 \%$ ethanol, air-dried, resuspended with $8 \mathrm{M}$ urea (Sigma) and $2 \times$ LDS loading buffer (Life Technologies) and boiled

Host cell culture. Human primary keratinocytes (HEKn, Gibco, C0015C) were cultured in DermaLife basal medium (Lifeline Cell Technology) supplemented with growth factors (DermaLife K LifeFactors kit, Lifeline Cell Technology) $1 \%$ fetal bovine serum (FBS, Gibco) and 1\% penicillin/streptomycin (Pen/Strep, Corning) and used up to passage 8 . THP-1 cells (ATCC, TIB-202) were cultured in RPMI 1640 medium containing L-glutamine (Corning) and supplemented with $10 \%$ FBS and $1 \%$ Pen/Strep and differentiated with phorbol 12-myristate 13-acetate (PMA, Sigma-Aldrich) at a final concentration of $1 \mu \mathrm{M}$ for $48 \mathrm{~h}$. The HEKn and THP-1 cells were authenticated by the manufacturers. Murine BMDMs were

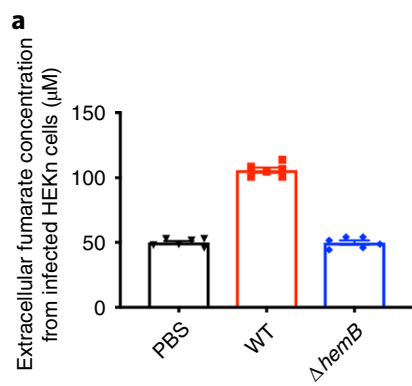

b

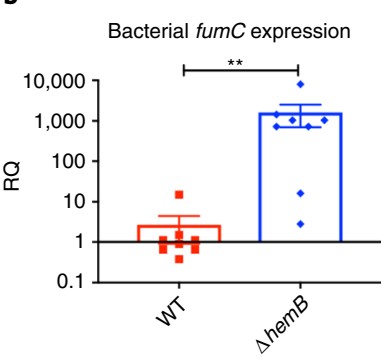

e

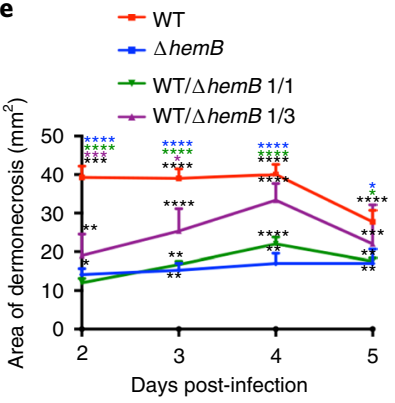

f

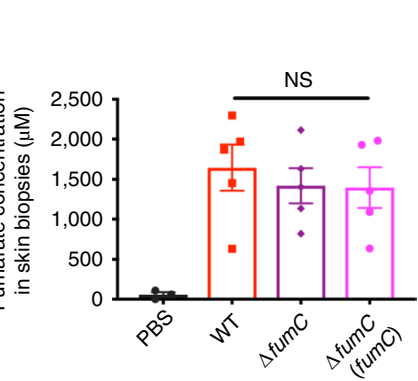

i

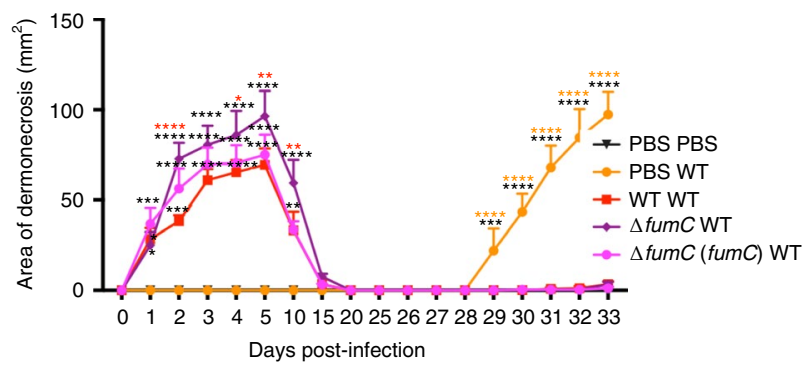

j

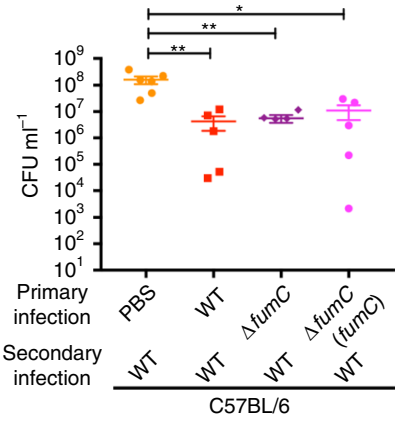

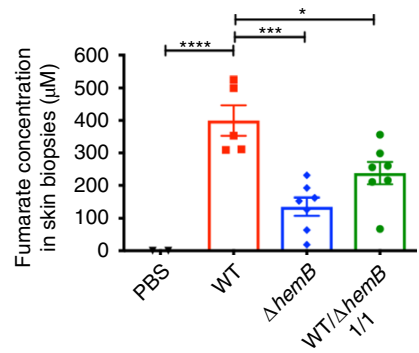

g

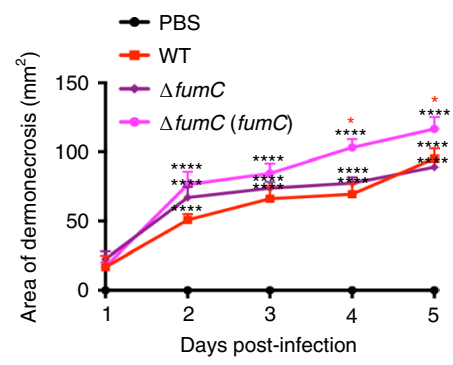

d

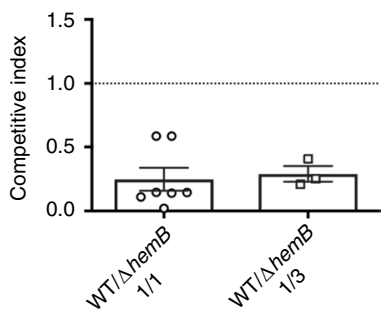

h

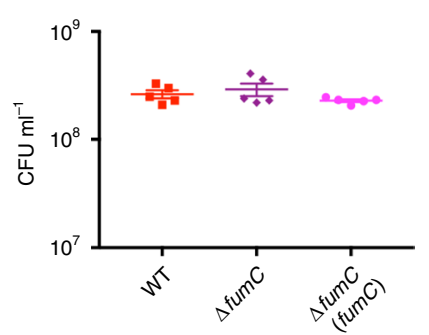

k

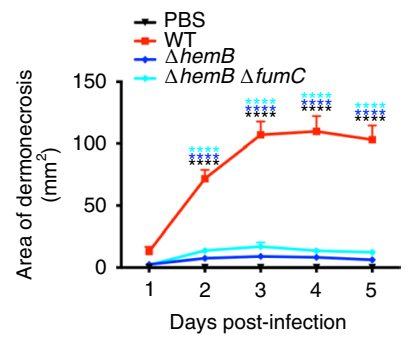

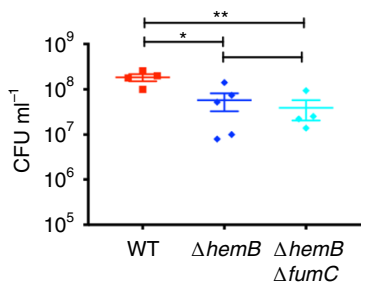

n

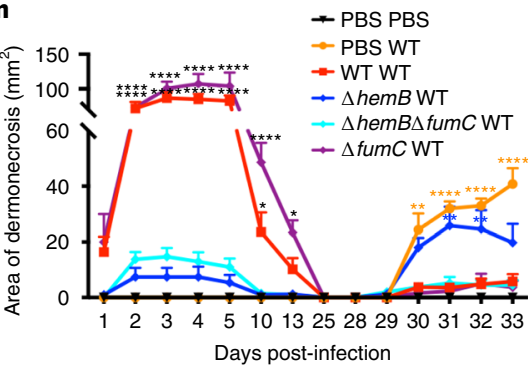

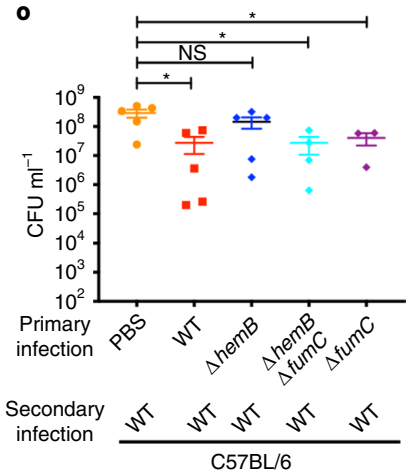


cultured in DMEM (Corning) supplemented with 1\% FBS with or without Pen/ Strep. All cell lines were routinely tested for mycoplasma contamination.

Infection of human primary keratinocytes and THP-1 cells. HEKn cells were seeded at $2 \times 10^{5}$ cells ml $^{-1}$ in 24 -well plates $24 \mathrm{~h}$ before infection and bacterial strains were cultured in liquid broth overnight. On the day of infection, all bacterial cultures were subinoculated 1:100 in liquid culture, except for the $\Delta$ hemB strains, and grown to an $\mathrm{OD}_{600 \mathrm{~nm}}$ of 1 . Fibronectin (Corning) was added to HEKn cells at $10 \mu \mathrm{g} \mathrm{ml}^{-1} 1 \mathrm{~h}$ before infection along with 2-DG (Sigma-Aldrich), BHA (Sigma-Aldrich), a pyruvate dehydrogenase inhibitor (CPI-613, Selleckchem), cytochalasin D (Sigma-Aldrich) and the autophagy inhibitors WM (Calbiochem), 3-MA (Sigma-Aldrich) and CQ (Sigma) at final concentrations of $100 \mathrm{mM}$, $200 \mu \mathrm{M}, 300 \mu \mathrm{M}, 20 \mu \mathrm{M}, 5 \mu \mathrm{M}, 20 \mu \mathrm{M}$ and $50 \mu \mathrm{M}$, respectively. The TLR2 agonists Pam3Cys (Invivogen) and lipoarabinomannan (Invivogen) were added at a final concentration of $10 \mu \mathrm{g} \mathrm{ml}^{-1} 1 \mathrm{~h}$ before infection for a total of $5 \mathrm{~h}$. Heat-killed $S$. aureus was prepared by treating bacteria at $65^{\circ} \mathrm{C}$ for $1 \mathrm{~h}$. HEKn cells were infected with the various $S$. aureus strains at a multiplicity of infection (m.o.i.) of 50 for 4 or $24 \mathrm{~h}$. For $24 \mathrm{~h}$ infections, lysostaphin was added $4 \mathrm{~h}$ post-infection at a final concentration of $10 \mu \mathrm{g} \mathrm{ml}^{-1}$

Transfection of human primary keratinocytes. Human Tlr2 and Tnfr 1 siRNAs (Dharmacon) and non-targeting control siRNAs (Santa Cruz) were reversetransfected for $24 \mathrm{~h}$ into HEKn cells using Lipofectamine RNAiMAX (Life Technologies) according to the manufacturer's instructions. Keratinocytes were transfected with poly dA:dT (InvivoGen) at a final concentration of $1 \mu \mathrm{g} \mathrm{ml}^{-1} \mathrm{using}$ Lipofectamine 3000 (Life Technologies) as per the manufacturer's protocol.

RNA extraction from mammalian cells. RNA was extracted from human keratinocytes using the E.Z.N.A Total RNA kit (Omega Bio-tek), followed by DNase treatment and reverse transcription as described above. The primer pairs P33/34 and P35/36 were used to amplify the genes coding for Aim2 and actin.

Lysostaphin protection assay. BMDMs were isolated from mice, differentiated in the presence of $20 \mathrm{ng} \mathrm{ml}^{-1}$ of murine M-CSF (Peprotech) and seeded at $1.15 \times 10^{6}$ cells $\mathrm{ml}^{-1}$ in DMEM (Corning) supplemented with $1 \%$ FBS. BMDMs were infected at an m.o.i. of 50 for $30 \mathrm{~min}$ and washed. Fresh DMEM containing 1\% FBS and $10 \mu \mathrm{g} \mathrm{ml}^{-1}$ lysostaphin (Sigma) was then added until the desired time point. The cells were washed, detached using TrypLE Express (Life Technologies), serially diluted and plated on BHI agar. Cell viability was determined by counting live cells using the trypan blue (Life Technologies) exclusion method ${ }^{44}$.

Extracellular flux analysis. HEKn cells were seeded at 30,000 cells per well in a Seahorse XF24 well plate (Agilent Technologies) and incubated at $37^{\circ} \mathrm{C}$ with $5 \% \mathrm{CO}_{2}$. A sensor cartridge was calibrated as per the manufacturer's instructions overnight at $37^{\circ} \mathrm{C}$ without $\mathrm{CO}_{2}$. On the day of infection, the primary keratinocytes were washed once in XF base medium (Agilent) supplemented with $2 \mathrm{mM}$ glutamine, and fibronectin was added at $10 \mu \mathrm{g} \mathrm{ml}^{-1} 1 \mathrm{~h}$ before infection. The cells were infected at an m.o.i. of 50 and incubated at $37^{\circ} \mathrm{C}$ without $\mathrm{CO}_{2}$ for $3 \mathrm{~h}$ The ECAR was measured using an XF24 analyser (Seahorse Bioscience). Each measurement cycle consisted of a mixing time of $3 \mathrm{~min}$ and a data acquisition period of $3 \mathrm{~min}$ ( 12 data points). Glucose was added at a final concentration of $10 \mathrm{mM}$ to stimulate glycolysis followed by the addition of oligomycin at $1 \mu \mathrm{M}$ to suppress oxidative phosphorylation and 2-DG at $50 \mathrm{mM}$ to inhibit glycolysis. Whenever THP-1 cells were used, they were seeded at a density of 150,000 cells per well and differentiated with PMA. The metabolic activity of the bacteria was determined by adding the same amount of bacteria in XF24 well plates without HEKn cells.

Metabolomics analysis. Human PBMCs were isolated from whole blood using Vacutainer CPT tubes (BD) according to the manufacturer's instructions. Following hypotonic lysis of red blood cells, the cells were seeded at a density of $1 \times 10^{6}$ cells $\mathrm{ml}^{-1}$ in XF base medium (Agilent) supplemented with $2 \mathrm{mM} \mathrm{L}$-glutamine for $3 \mathrm{~h}$. Glucose was then added at a final concentration of $10 \mathrm{mM}$ for $30 \mathrm{~min}$. Tissue culture supernatants were then saved for metabolite extraction. A 50\% methanol solution $(\mathrm{v} / \mathrm{v})$ was used to extract metabolites from tissue culture supernatants. High-resolution mass spectrometry data were acquired on the Exactive Mass Spectrometer (Thermo Fisher) in negative mode using 25 min reverse phase gradients and ion-pairing chromatography at the University of Calgary. Metabolites were identified using the known chromatographic retention times of standards, and metabolite signals were quantified using MAVEN $^{45}$. The extracellular metabolites detected from PBMCs are listed in Supplementary Table 4.

THP- 1 cells were seeded at a density of $3 \times 10^{6}$ cells per well in a 6-well tray and differentiated with PMA $48 \mathrm{~h}$ before infection. On the day of infection, the cells were washed once and infected at an m.o.i. of 50 in XF base medium (Agilent) supplemented with $2 \mathrm{mM}$ L-glutamine for $3 \mathrm{~h}$. Glucose was then added at a final concentration of $10 \mathrm{mM}$ for $30 \mathrm{~min}$. Tissue culture supernatants were then saved for metabolite extraction and the cells were washed once in Milli-Q water before lysis using liquid nitrogen. A methanol/chloroform solution $(9: 1 \mathrm{v} / \mathrm{v})$ containing $0.5 \mathrm{nM}^{13} \mathrm{C}$-sorbitol and $5 \mathrm{nM}{ }^{13} \mathrm{C},{ }^{15} \mathrm{~N}$-valine as internal standards was used to extract metabolites from cell lysates, and tissue culture supernatants. Samples were processed at Metabolomics Australia (Bio21, The University of Melbourne, Australia) on a gas chromatography-triple-quadrupole mass spectrometer. Metabolite detection was performed using the Smart Metabolites Database (Shimadzu). The Automatic Adjustment of Retention in GCMSsolution software (version 4.42 Shimadzu) and a standard alkane series mixture (C7-C33, Restek) were used to correct retention time shifts in the acquisition method. Annotated metabolites were quantified. To determine the relationship between samples, partial least-squares discriminant analysis ${ }^{46}$ was computed using the mixOmics $\mathrm{R}$ package $^{47}$. Multivariate analysis plots were generated using the $\mathrm{R}$ function plotIndiv (Plot Of Individuals), with the ellipses representing 95\% confidence intervals of the sample space. The relative abundance of each metabolite was log-transformed and the median of all metabolites from each sample was then calculated. Metabolites from each sample were then normalized to the median to account for any biomass variability and plotted as a heatmap using GraphPad Prism Version 7.00 (GraphPad). The intracellular and extracellular metabolites from THP-1 cells are listed in Supplementary Tables 5 and 6, respectively.

Western blotting. Proteins isolated from bacterial cultures were separated by gel electrophoresis, transferred to PVDF membranes (Invitrogen) and probed with anti-Hla (Sigma), anti-LukA, anti-LukE and anti-LukD (gift from V. J. Torres) at concentrations of 1:5,000, 1:5,000, 1:10,000 and 1:7,500, respectively.

HEKn cells were lysed in RIPA lysis buffer ( $1 \mathrm{mM}$ Tris-HCl, pH 7.5, $15 \mathrm{mM}$ $\mathrm{NaCl}, 0.5 \mathrm{mM}$ EDTA, $0.01 \%$ SDS, $0.1 \%$ Triton X-100, 0.1\% deoxycholate) containing $1 \times$ HALT protease inhibitor cocktail (ThermoFisher Scientific). Protein concentrations were quantified using the Precision Red advanced protein kit (Cytoskeleton) and samples were standardized to a concentration of $100 \mu \mathrm{g}$. The samples were subjected to gel electrophoresis and the gels (Invitrogen) were transferred to PVDF membranes (Invitrogen) using the iBlot machine (LifeTechnologies). Antibodies against PMLKL, MLKL, PS6, TNFR1 (Cell Signaling), TLR2 (EMD Millipore) and LC3B, $\beta$-actin (Sigma-Aldrich) were used according to the manufacturer's instructions. Secondary antibodies conjugated to horseradish peroxidase (Abcam, Santa Cruz) were diluted 1:10,000. Images were visualized using a digital chemiluminescent detection imager (ProteinSimple) and ImageJ.

Mitochondrial ROS and membrane potential measurement. HEKn cells were infected at an m.o.i. of 50 for $3 \mathrm{~h}$. Cells were stimulated with TBHP (Life Technologies) at a final concentration of $400 \mu \mathrm{M}$ for $1 \mathrm{~h}$. The cells were washed, detached with TrypLE Express (Life Technologies) and stained for mitochondrial membrane potential (Dilc1(5), Invitrogen), cell viability (LIVE/DEAD stain, Invitrogen) and mitochondrial ROS (MitoSox, Invitrogen) for $20 \mathrm{~min}$ at $37^{\circ} \mathrm{C}$ in DermaLife medium. The cells were washed three times with PBS and analysed using a FACS CantoII flow cytometer machine.

Mice. Male and female mice were purchased from Jackson Laboratories (C57BL/6; stock number 000664, Il1r1 ${ }^{-/-}$; stock number 003245, Aim2 $2^{-/-}$; stock number 029472, Rag2 KO; stock number 008449). $\mathrm{Mlkl}^{-/-}$mice $^{14,48}$ were obtained from J. Silke (WEHI Australia) via D. R. Green (St Jude Children's Research Hospital) and Ripk1d (Ripk1 $1^{K 45 A}$ ) mice ${ }^{49,50}$ were obtained from S. B. Berger from GlaxoSmithKline. The animal work protocol (AAAR5412) was approved by the Institutional Animal Care and Use Committee. Animal experiments were carried out in strict accordance with the recommendations in the Guide for the Care and Use of Laboratory Animals of the NIH, the Animal Welfare Act, and US federal law. Mice were aged 6-8 weeks at the start of the experiment.

Mouse infection. Mice were infected intradermally on the back with $1 \times 10^{7}$ CFUs of $S$. aureus $8325-4$ or the isogenic $\Delta$ hemB mutant in $100 \mu \mathrm{l}$ PBS or $2 \times 10^{6}$ CFUs of $S$. aureus USA300 LAC or the $\Delta$ hemB, $\Delta$ hemB $\Delta$ fumC or $\Delta$ fumC mutants or the complemented strains in $100 \mu \mathrm{l}$ PBS. PBS alone was used as a control. For trained immunity experiments, mice were challenged with the WT strain 28 days following primary infection at the same site. Lesions were measured and punch biopsies $(5 \mathrm{~mm}$ ) were taken in the middle of the infected lesion. The biopsied tissue was homogenized through $40 \mu \mathrm{m}$ cell strainers (Falcon) before the hypotonic lysis of red blood cells. The homogenates were serially diluted and plated. Bacterial counts were enumerated. Keratinocytes and immune cells were spun down at $700 \mathrm{~g}$ at $4{ }^{\circ} \mathrm{C}$ and the supernatants were used for cytokine analysis. Immune cells were stained with fluorescently conjugated markers and analysed by flow cytometry. No calculation was used to determine the number of mice required. No data blinding was performed.

Flow cytometry measurement. Human keratinocytes were stained with LIVE/ DEAD stain (Invitrogen) for $20 \mathrm{~min}$ at $4{ }^{\circ} \mathrm{C}$, washed and permeabilized with permeabilization buffer $(0.05 \%$ saponin, $2 \%$ FBS in PBS) for $10 \mathrm{~min}$ at room temperature. The cells were then stained with anti-Aim2 antibody (Abcam) for $30 \mathrm{~min}$ at $4^{\circ} \mathrm{C}$, washed and stained with secondary antibodies before fixing with 2\% paraformaldehyde (Electron Microscopy Sciences).

Red blood cells were lysed from mouse skin homogenates and the remaining cells were washed with FACS buffer (10\% FBS and $0.1 \%$ sodium 
azide in PBS). Cells were stained for $30 \mathrm{~min}$ at $4{ }^{\circ} \mathrm{C}$ in the presence of counting beads (Bangs Laboratories) and labelled with a combination of BV605labelled anti-CD11c (Biolegend), BV421-lalbelled CD86 (Biolegend), BV510labelled CD103 (Biolegend), PE-CF594-labelled anti-Ly6C (BD Horizon), PerCP-Cy5.5-labelled anti-Ly6G (Biolegend), BV650-labelled anti-NK-1.1 (Biolegend), AF700-labelled CD45 (Biolegend), APC/Cy7-labelled MHCII (Biolegend), PE-labelled CD207 (Biolegend), AF594-labelled anti-CD11b (BioLegend) and Fc block (Biolegend). Analysis of immune cell populations was conducted using BD LSR II (BD Biosciences). Cells in the skin were classified as follows: Langerhans cells, $\mathrm{CD}_{4} 5^{+} \mathrm{CD} 11 \mathrm{~b}^{+} \mathrm{CD} 11 \mathrm{c}^{+} \mathrm{MHCII}{ }^{+} \mathrm{CD} 207^{+}$; Langerhans DCs, CD $45^{+} \mathrm{CD} 11 \mathrm{~b}^{+} \mathrm{CD} 11 \mathrm{c}^{+} \mathrm{MHCII}{ }^{+} \mathrm{CD} 207^{+} \mathrm{CD} 103$

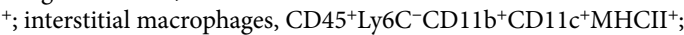

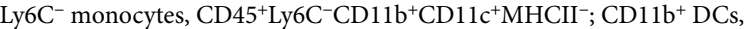

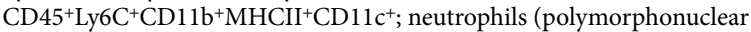

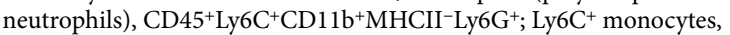

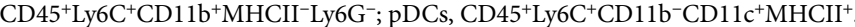
All flow cytometry data were analysed on FlowJo version 10

Cytokine analysis. Supernatants from in vivo tissue homogenates were collected and cytokine concentrations were quantified by Eve Technologies (Calgary, Canada).

Quantification of fumarate. Fumarate quantification was performed using the fumarate assay kit (Abcam) according to the manufacturer's instructions.

Statistical analysis. Samples without a normal distribution were analysed using the non-parametric Mann-Whitney test. Samples with normal distribution were analysed using one- or two-way analysis of variance (ANOVA). $P<0.05$ between groups was considered significant. Statistical analysis was performed using GraphPad Prism Version 7.00 (GraphPad).

Reporting Summary. Further information on research design is available in the Nature Research Reporting Summary linked to this article.

\section{Data availability}

Whole genome sequence data of the $\Delta h e m B, \Delta$ fumC and $\Delta h e m B \Delta f u m C$ LAC mutants and the complemented strains $\Delta$ hemB (hemB) and $\Delta$ fumC (fumC) were submitted to the European Nucleotide Archive under BioProject number PRJEB30093, accession numbers ERS3011129, ERS3011131, ERS3398722, ERS3011130 and ERS3011132, respectively. Data that support the findings of this study are available from the corresponding author upon request.

Received: 30 May 2019; Accepted: 19 September 2019; Published online: 4 November 2019

\section{References}

1. Kahl, B. C., Becker, K. \& Loffler, B. Clinical significance and pathogenesis of staphylococcal small colony variants in persistent infections. Clin. Microbiol. Rev. 29, 401-427 (2016).

2. Kennedy, A. D. et al. Targeting of alpha-hemolysin by active or passive immunization decreases severity of USA300 skin infection in a mouse model. J. Infect. Dis. 202, 1050-1058 (2010).

3. Kernodle, D. S., Voladri, R. K., Menzies, B. E., Hager, C. C. \& Edwards, K. M. Expression of an antisense hla fragment in Staphylococcus aureus reduces alpha-toxin production in vitro and attenuates lethal activity in a murine model. Infect. Immun. 65, 179-184 (1997).

4. Kobayashi, S. D. et al. Comparative analysis of USA300 virulence determinants in a rabbit model of skin and soft tissue infection. J. Infect. Dis. 204, 937-941 (2011).

5. Menzies, B. E. \& Kernodle, D. S. Site-directed mutagenesis of the alpha-toxin gene of Staphylococcus aureus: role of histidines in toxin activity in vitro and in a murine model. Infect. Immun. 62, 1843-1847 (1994).

6. Proctor, R. A. \& Peters, G. Small colony variants in staphylococcal infections: diagnostic and therapeutic implications. Clin. Infect. Dis. 27, 419-422 (1998).

7. Proctor, R. A. et al. Small colony variants: a pathogenic form of bacteria that facilitates persistent and recurrent infections. Nat. Rev. Microbiol. 4 295-305 (2006).

8. Kriegeskorte, A. et al. Staphylococcus aureus small colony variants show common metabolic features in central metabolism irrespective of the underlying auxotrophism. Front. Cell. Infect. Microbiol. 4, 141 (2014).

9. Proctor, R. A. et al. Staphylococcus aureus small colony variants (SCVs): a road map for the metabolic pathways involved in persistent infections. Front. Cell. Infect. Microbiol. 4, 99 (2014).

10. Tuchscherr, L. et al. Staphylococcus aureus small-colony variants are adapted phenotypes for intracellular persistence. J. Infect. Dis. 202, 1031-1040 (2010).

11. Arts, R. J., Joosten, L. A. \& Netea, M. G. Immunometabolic circuits in trained immunity. Semin. Immunol. 28, 425-430 (2016).
12. O’Neill, L. A., Kishton, R. J. \& Rathmell, J. A guide to immunometabolism for immunologists. Nat. Rev. Immunol. 16, 553-565 (2016).

13. Wickersham, M. et al. Metabolic stress drives keratinocyte defenses against Staphylococcus aureus infection. Cell Rep. 18, 2742-2751 (2017).

14. Kitur, K. et al. Necroptosis promotes Staphylococcus aureus clearance by inhibiting excessive inflammatory signaling. Cell Rep. 16, 2219-2230 (2016)

15. Baek, K. T. et al. Genetic variation in the Staphylococcus aureus 8325 strain lineage revealed by whole-genome sequencing. PLoS ONE 8, e77122 (2013)

16. Herbert, S. et al. Repair of global regulators in Staphylococcus aureus 8325 and comparative analysis with other clinical isolates. Infect. Immun. 78, 2877-2889 (2010)

17. Horsburgh, M. J. et al. $\sigma^{\mathrm{B}}$ modulates virulence determinant expression and stress resistance: characterization of a functional $r s b U$ strain derived from Staphylococcus aureus 8325-4. J. Bacteriol. 184, 5457-5467 (2002).

18. Giachino, P., Engelmann, S. \& Bischoff, M. $\varsigma^{\mathrm{B}}$ activity depends on RsbU in Staphylococcus aureus. J. Bacteriol. 183, 1843-1852 (2001).

19. Anzaldi, L. L. \& Skaar, E. P. Overcoming the heme paradox: heme toxicity and tolerance in bacterial pathogens. Infect. Immun. $\mathbf{7 8}$ 4977-4989 (2010)

20. Atalla, H., Gyles, C. \& Mallard, B. Staphylococcus aureus small colony variants (SCVs) and their role in disease. Anim. Health Res. Rev. 12, 33-45 (2011).

21. von Eiff, C. et al. A site-directed Staphylococcus aureus hemB mutant is a small-colony variant which persists intracellularly. J. Bacteriol. 179, 4706-4712 (1997)

22. Palma Medina, L. M. et al. Metabolic cross-talk between human bronchial epithelial cells and internalized Staphylococcus aureus as a driver for infection. Mol. Cell. Proteom. 18, 892-908 (2019).

23. LaRocca, T. J., Sosunov, S. A., Shakerley, N. L., Ten, V. S. \& Ratner, A. J. Hyperglycemic conditions prime cells for RIP1-dependent Necroptosis. J. Biol. Chem. 291, 13753-13761 (2016).

24. McCaig, W. D. et al. Hyperglycemia potentiates a shift from apoptosis to RIP1-dependent necroptosis. Cell Death Discov. 4, 55 (2018).

25. Cai, Z. et al. Plasma membrane translocation of trimerized MLKL protein is required for TNF-induced necroptosis. Nat. Cell Biol. 16, 55-65 (2014).

26. Chen, X. et al. Translocation of mixed lineage kinase domain-like protein to plasma membrane leads to necrotic cell death. Cell Res. 24, 105-121 (2014).

27. Gonzalez-Juarbe, N. et al. Pore-forming toxins induce macrophage necroptosis during acute bacterial pneumonia. PLoS Pathog. 11, e1005337 (2015).

28. Kitur, K. et al. Toxin-induced necroptosis is a major mechanism of Staphylococcus aureus lung damage. PLoS Pathog. 11, e1004820 (2015).

29. Vitko, N. P., Spahich, N. A. \& Richardson, A. R. Glycolytic dependency of high-level nitric oxide resistance and virulence in Staphylococcus aureus. mBio 6, e00045-15 (2015).

30. Yang, Z. et al. RIP3 targets pyruvate dehydrogenase complex to increase aerobic respiration in TNF-induced necroptosis. Nat. Cell Biol. 20, 186-197 (2018).

31. Kucera, O. et al. The effect of tert-butyl hydroperoxide-induced oxidative stress on lean and steatotic rat hepatocytes in vitro. Oxid. Med. Cell. Longev. 2014, 752506 (2014)

32. Galluzzi, L., Kepp, O., Chan, F. K. \& Kroemer, G. Necroptosis: mechanisms and relevance to disease. Annu. Rev. Pathol. 12, 103-130 (2017).

33. Goodall, M. L. et al. The autophagy machinery controls cell death switching between apoptosis and necroptosis. Dev. Cell 37, 337-349 (2016).

34. Bhattacharyya, S. et al. Small colony variants of Staphylococcus aureus isolated from a patient with infective endocarditis: a case report and review of the literature. Iran. J. Microbiol. 4, 98-99 (2012).

35. Jonsson, I. M. et al. Virulence of a hemB mutant displaying the phenotype of a Staphylococcus aureus small colony variant in a murine model of septic arthritis. Microb. Pathog. 34, 73-79 (2003).

36. Chan, L. C. et al. Innate immune memory contributes to host defense against recurrent skin and skin structure infections caused by methicillin-resistant staphylococcus aureus. Infect. Immun. 85, e00876-16 (2017)

37. Chan, L. C. et al. Protective immunity in recurrent Staphylococcus aureus infection reflects localized immune signatures and macrophage-conferred memory. Proc. Natl Acad. Sci. USA 115, E11111-E11119 (2018).

38. van der Heijden, C. et al. Epigenetics and trained immunity. Antioxid. Redox Signal. 29, 1023-1040 (2018).

39. Arts, R. J. et al. Glutaminolysis and fumarate accumulation integrate immunometabolic and epigenetic programs in trained immunity. Cell Metab. 24, 807-819 (2016).

40. Kornberg, M. D. et al. Dimethyl fumarate targets GAPDH and aerobic glycolysis to modulate immunity. Science 360, 449-453 (2018).

41. Naik, S. et al. Inflammatory memory sensitizes skin epithelial stem cells to tissue damage. Nature 550, 475-480 (2017); erratum 560, E2 (2018).

42. Monk, I. R., Tree, J. J., Howden, B. P., Stinear, T. P. \& Foster, T. J. Complete bypass of restriction systems for major Staphylococcus aureus lineages. mBio 6, e00308-e00315 (2015).

43. Monk, I. R., Howden, B. P., Seemann, T. \& Stinear, T. P. Correspondence: Spontaneous secondary mutations confound analysis of the essential two-component system WalKR in Staphylococcus aureus. Nat. Commun. 8, 14403 (2017). 
44. Strober, W. Trypan blue exclusion test of cell viability. Curr. Protoc. Immunol. 111, A3.B.1-A3.B.3 (2015).

45. Melamud, E., Vastag, L. \& Rabinowitz, J. D. Metabolomic analysis and visualization engine for LC-MS data. Anal. Chem. 82, 9818-9826 (2010).

46. Barker, M. \& Rayens, W. Partial least squares for discrimination. J. Chemom. 17, 166-173 (2003).

47. Rohart, F., Gautier, B., Singh, A. \& Le Cao, K. A. mixOmics: an R package for 'omics feature selection and multiple data integration. PLoS Comput. Biol. 13, e1005752 (2017)

48. Murphy, J. M. et al. The pseudokinase MLKL mediates necroptosis via a molecular switch mechanism. Immunity 39, 443-453 (2013).

49. Berger, S. B. et al. Cutting edge: RIP1 kinase activity is dispensable for normal development but is a key regulator of inflammation in SHARPINdeficient mice. J. Immunol. 192, 5476-5480 (2014).

50. Soong, G. et al. Methicillin-resistant Staphylococcus aureus adaptation to human keratinocytes. mBio 6, e00289-15 (2015).

\section{Acknowledgements}

We thank I. Lewis (University of Calgary), R. Groves (University of Calgary) and D. P. De Souza (Metabolomics Australia) for their support with the metabolomics studies and V. J. Torres (New York University School of Medicine) for his gift of antibodies against staphylococci toxins. This work was supported by NIH grant R01AI103854 to A.P., NIH grant S10RR027050 to the Columbia Center for Translational Immunology Flow Cytometry Core and NHMRC grant (Australia) APP1066791 to B.P.H.

\section{Author contributions}

T.W.F.L. and A.P. conceived the project, designed the experiments and interpreted the data. T.W.F.L. performed the in vitro and in vivo experiments and data analysis. I.R.M. constructed the bacterial mutants and contributed to the transcriptomics studies. K.P.A. contributed to the in vitro and in vivo work pertaining to Aim2. N.W. performed the in vitro THP-1 infection and A.M. analysed the metabolomics data. S.A.R., S.P., L.P.N. F.D. and S.J.G. assisted with the in vitro and in vivo experiments and data analysis. T.W.F.L., B.P.H. and A.P. wrote and edited the paper.

\section{Competing interests}

The authors declare no competing interests.

\section{Additional information}

Extended data is available for this paper at https://doi.org/10.1038/s41564-019-0597-0. Supplementary information is available for this paper at https://doi.org/10.1038/ s41564-019-0597-0.

Correspondence and requests for materials should be addressed to A.P.

Reprints and permissions information is available at www.nature.com/reprints. Publisher's note Springer Nature remains neutral with regard to jurisdictional claims in published maps and institutional affiliations.

(c) The Author(s), under exclusive licence to Springer Nature Limited 2019 
A

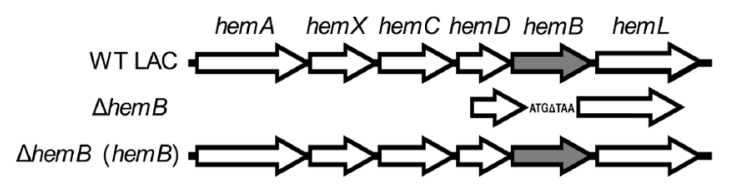

C

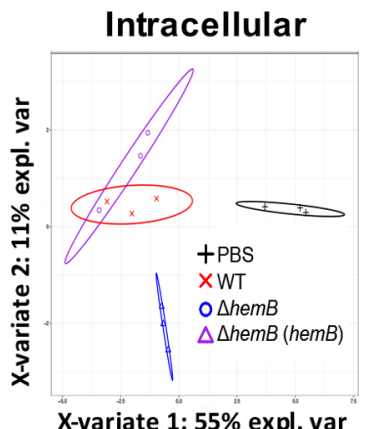

X-variate 1: 55\% expl. var

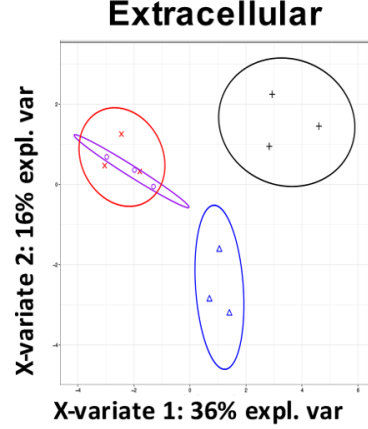

E

\section{Extracellular THP-1}

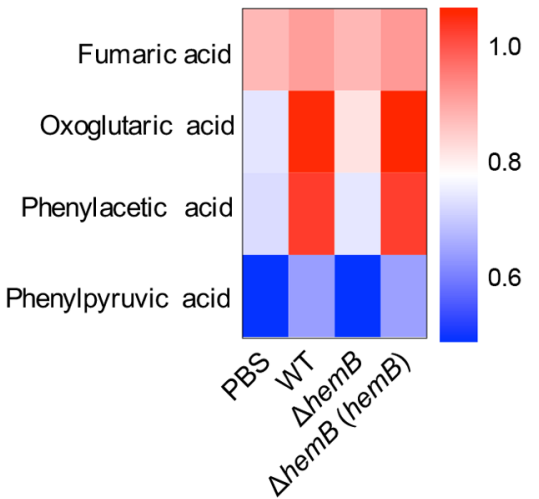

F

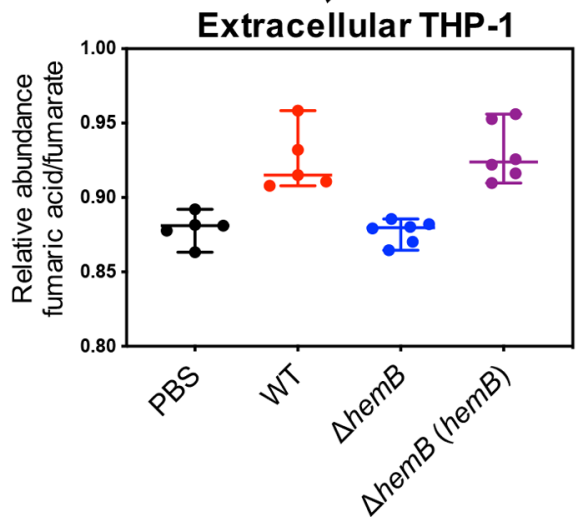

B

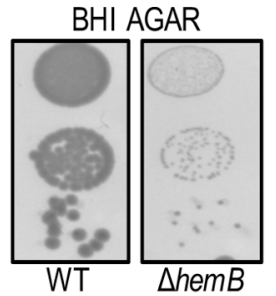

$\mathrm{D}$

\section{Intracellular THP-1}

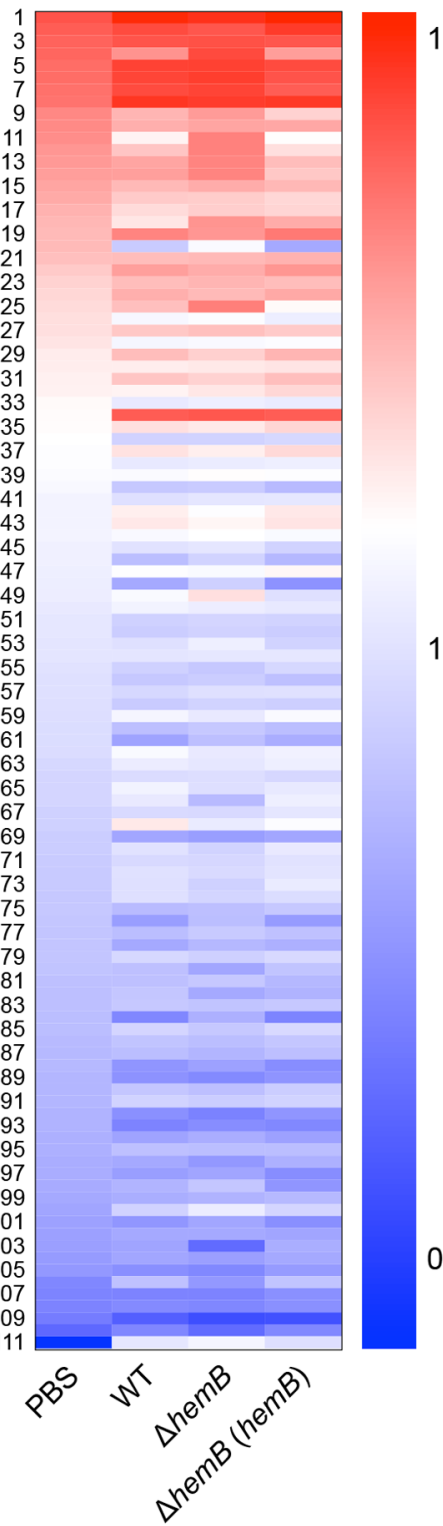

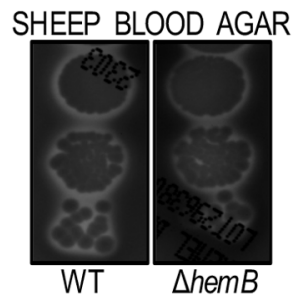

\section{Extracellular THP-1}

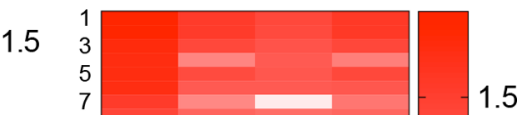

1.5

Extended data 1 | $\Delta$ hemB LAC SCVs stimulate a distinct metabolic response in THP-1 cells. (A) Construction of the $\triangle$ hemB mutant in the USA300 LAC background. (B) White light image of WT LAC and the $\Delta$ hemB LAC mutant on BHI and blood agar plates to better illustrate pinpoint size of the $\Delta$ hemB $\mathrm{LAC}$ mutant on $\mathrm{BHI}$ agar and $\beta$ (complete)-hemolysis on blood agar, image shown is from 1 representative experiment, $\mathrm{n}=3$ independent experiments. (C) Representative PCA score plots showing principal component 1 (PC1/variate 1) versus PC2/variate 2 of the total intracellular and extracellular THP-1 metabolomes, $n=2$ independent experiments in triplicate. (D) Heatmaps of the total intracellular and extracellular THP-1 metabolomes, $n=2$. (E) Heatmap of lower abundance metabolites in the extracellular THP-1 metabolomes, $n=2$. (F) Relative quantification of fumarate in the extracellular metabolome of uninfected and infected THP-1 cells. Data represent median with $95 \% \mathrm{Cl}, \mathrm{n}=2$ independent experiments in triplicate.. 
A

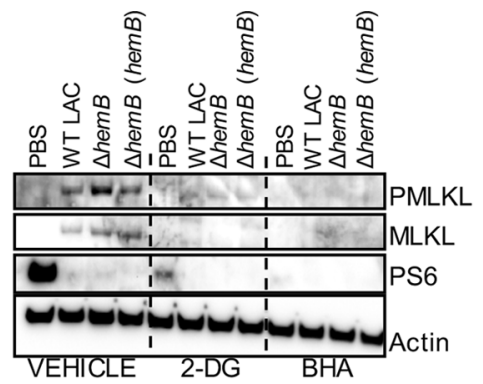

C

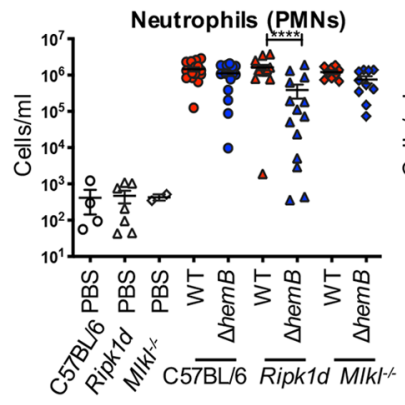

CD11b+ DCs

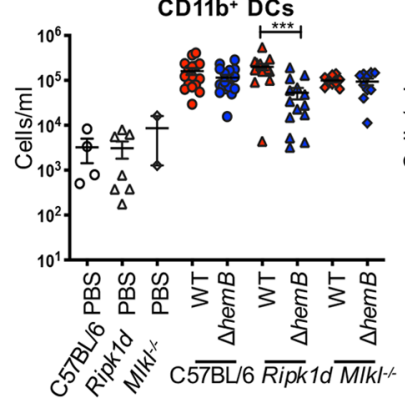

D

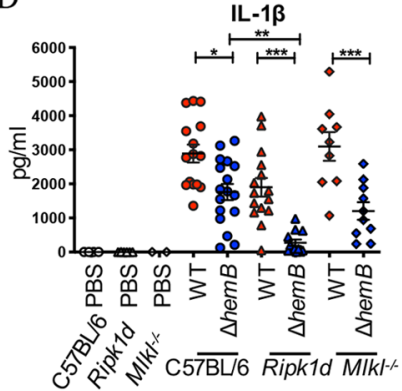

MIG

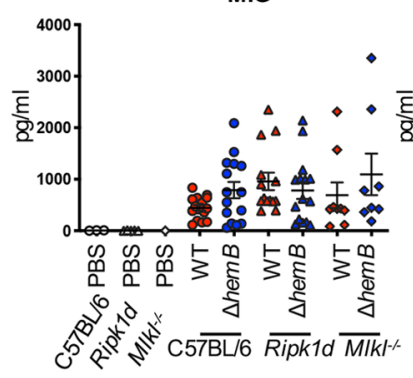

Interstitial macrophages

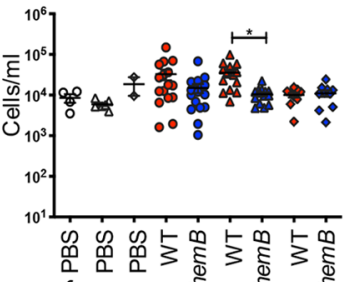

की की

Langerhans DCs

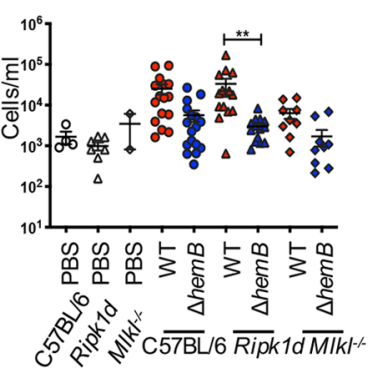

C仓

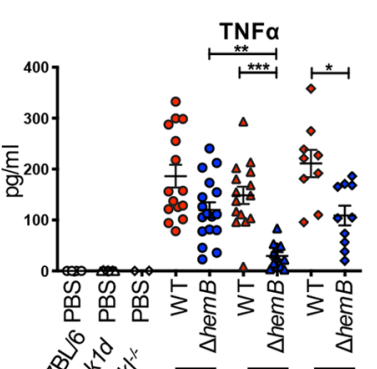

की

$\mathrm{KC}$

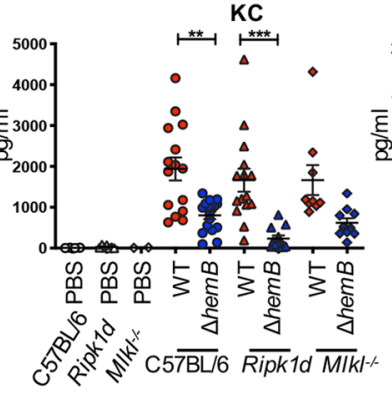

B

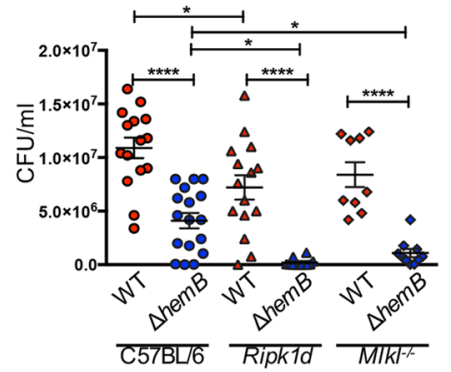

Ly6C- monocytes
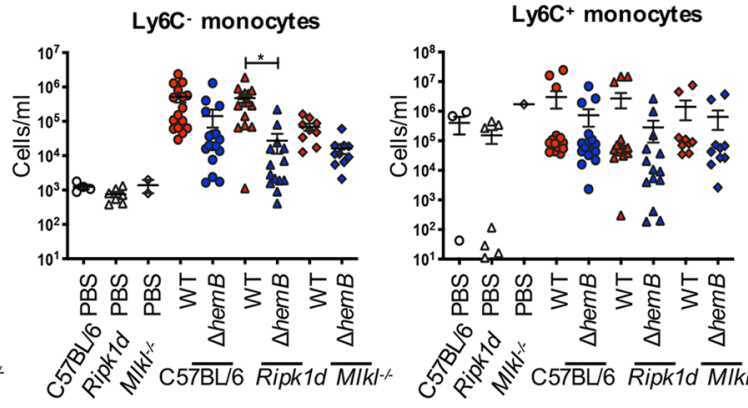

की

Langerhans cells

Plasmacytoid DCs (pDCs)
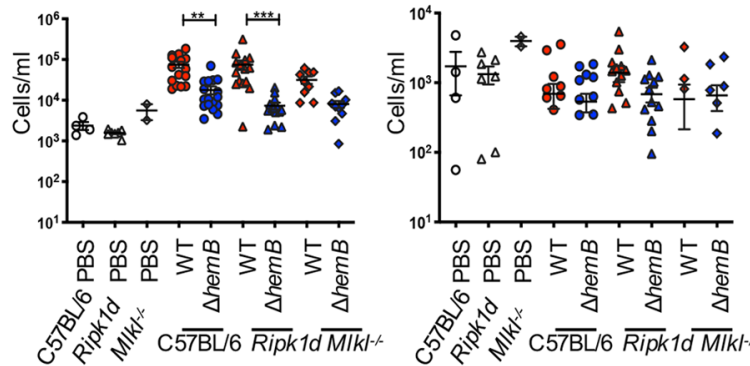

so

co

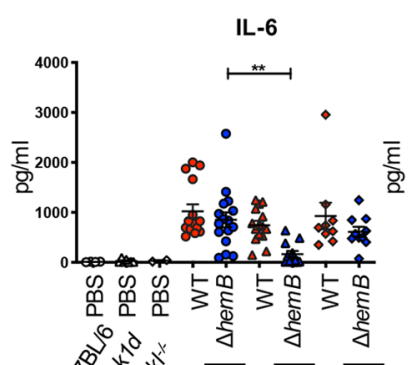

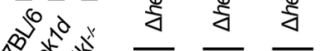

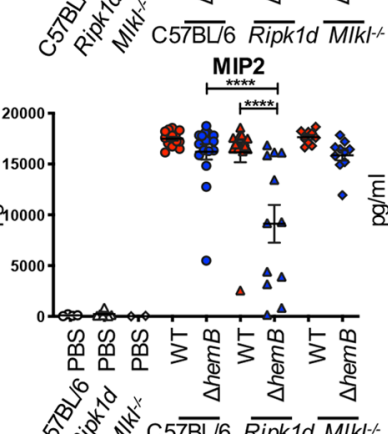

IFNy

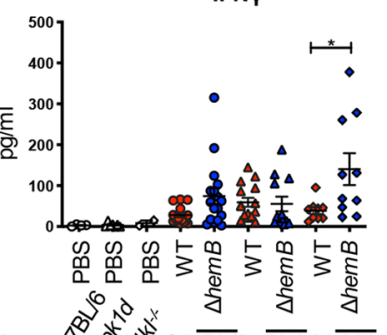

कि

LIX

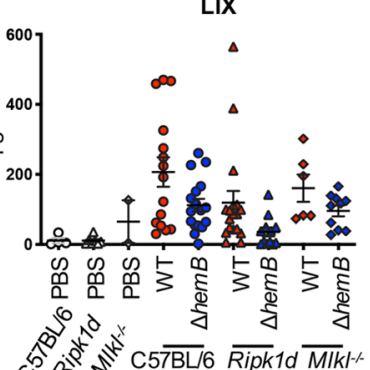

Extended data 2 | Necroptosis promotes $\Delta$ hemB SCV persistence. (A) A representative immunoblot showing the induction of PMLKL and PS6 in HEKn cells exposed to PBS, WT LAC, $\Delta$ hemB LAC or the complemented strain for $4 \mathrm{~h}$ in the presence and absence of 2-DG or BHA, $\mathrm{n}=3$ independent experiments. (B) Bacterial load in skin biopsies 5 days following infection of WT C57BL/6 or Ripk1d or Mlk/-1- mice with WT 8325-4 and $\Delta$ hemB 8325-4. (C) Innate immune cell recruitment from (B) at day 5. (D) Cytokine measurements from mouse skin biopsies from (B) at day 5 For (B-D), data represent mean \pm SEM, $\mathrm{n}=15$ (C57BL/6 WT), $\mathrm{n}=17$ (C57BL/6 $\Delta$ hemB). $\mathrm{n}=15$ (Ripk1d WT), $\mathrm{n}=12\left(\right.$ Ripk1d $\Delta$ hemB), $\mathrm{n}=9\left(\mathrm{Mlkl}{ }^{-/-} \mathrm{WT}\right)$ or $\mathrm{n}=10\left(\mathrm{Mlkl} \mathrm{I}^{-/-} \Delta\right.$ hemB) independent animals. Statistical analysis was performed by one-way ANOVA, ${ }^{\star \star \star \star} p<0.0001,{ }^{\star \star \star} p<0.001,{ }^{\star \star} p<0.01,{ }^{\star} p<0.05$.. 
A

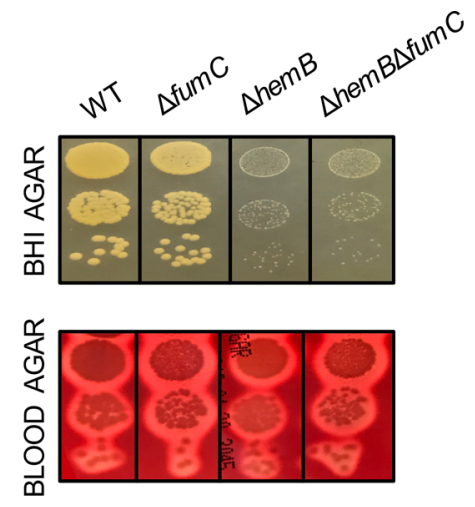

$\mathrm{C}$

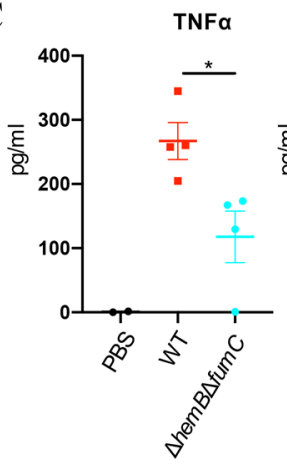

B
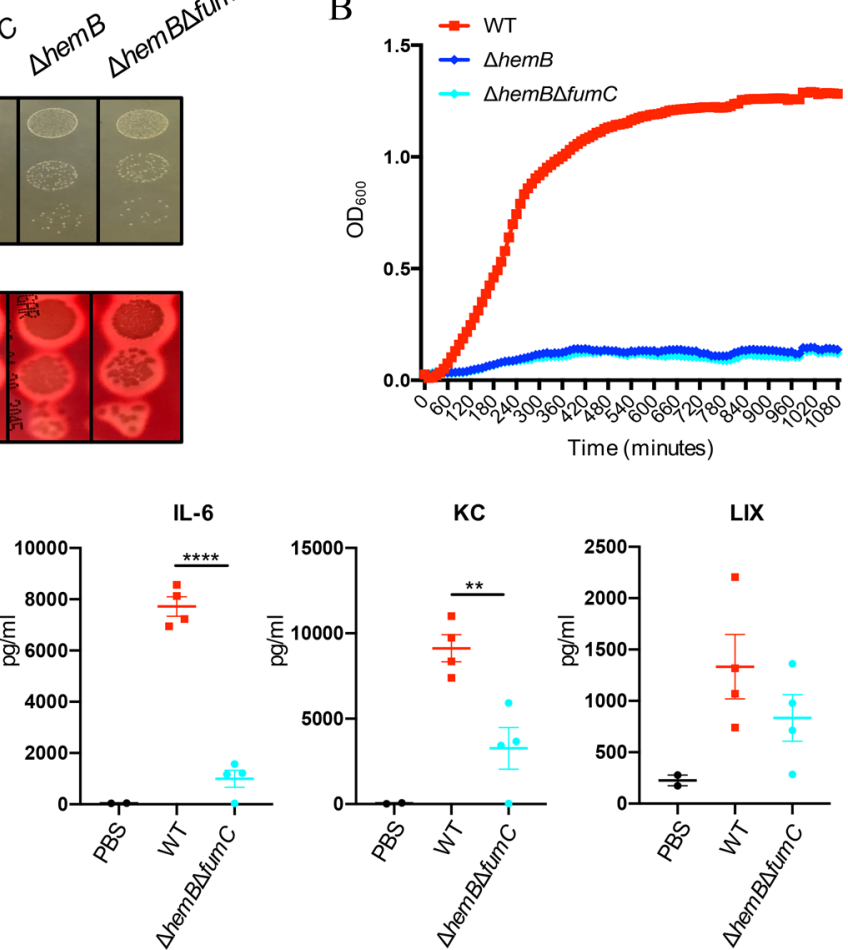

KC

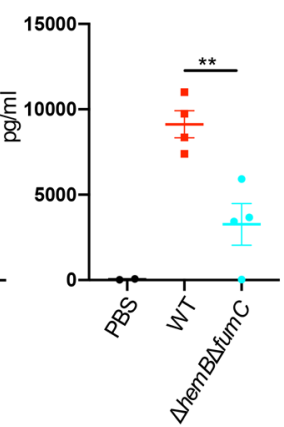

LIX

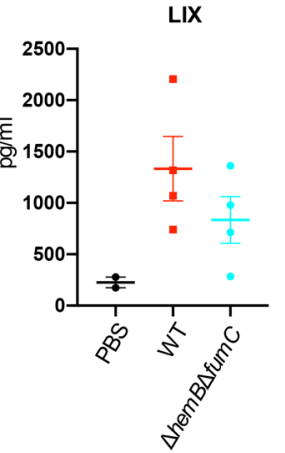

Extended data 3 | Characterization of the $\Delta$ hemB $\Delta$ fumC double mutant. (A) Growth of the $\Delta$ hemB $\Delta$ fumC double mutant as compared to WT LAC, the $\Delta$ hemB or $\Delta$ fumC single mutants on $\mathrm{BHI}$ and blood agar plates. Various dilutions of bacterial cultures were plated. Image shown is from 1 representative experiment, $\mathrm{n}=3$ independent experiments. (B) Growth of the $\Delta$ hemB $\Delta$ fumC double mutant as compared to WT LAC and the $\Delta$ hemB single mutant in liquid LB culture. $n=3$ independent experiments. (C) Cytokine measurements from the skin of mice 5 days post infection with WT LAC or the $\Delta$ hemB $\Delta$ fumC mutant. Data represent mean $\pm \mathrm{SEM}, \mathrm{n}=4$ independent animal samples.. Statistical analysis was performed by one-way ANOVA, ${ }^{\star \star \star \star} \mathrm{p}<$ $0.0001,{ }^{\star \star \star} p<0.001,{ }^{\star \star} p<0.01,{ }^{\star} p<0.05$. 
A

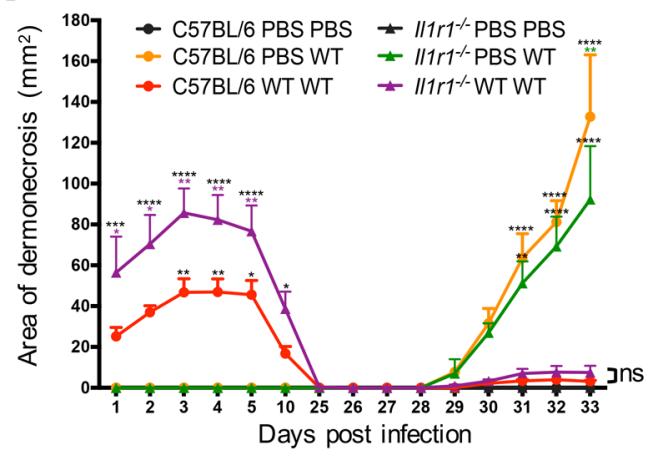

D

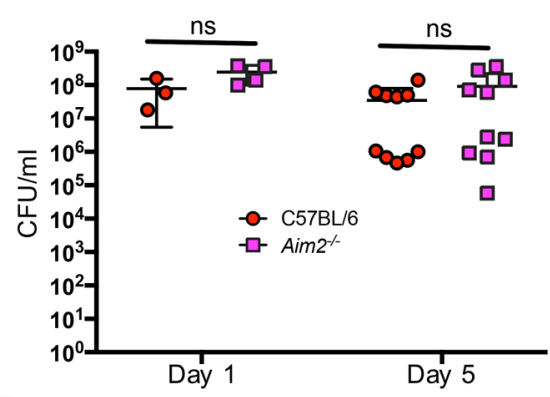

F

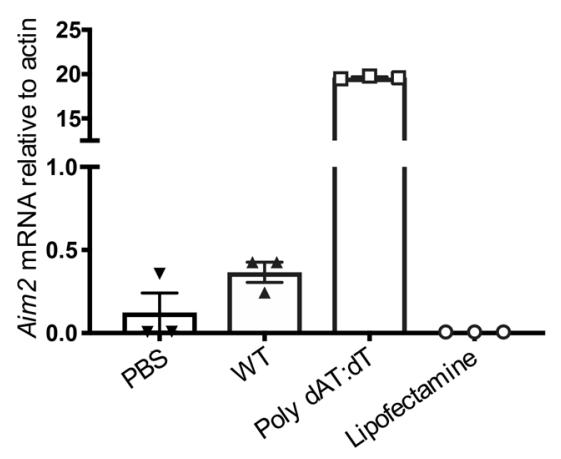

B

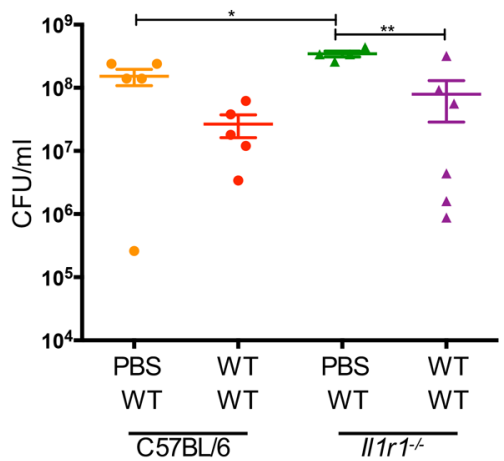

E

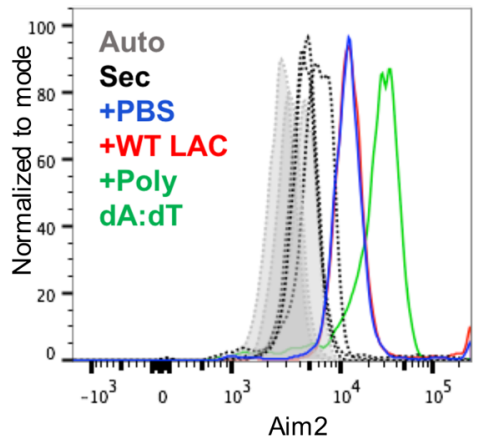

C
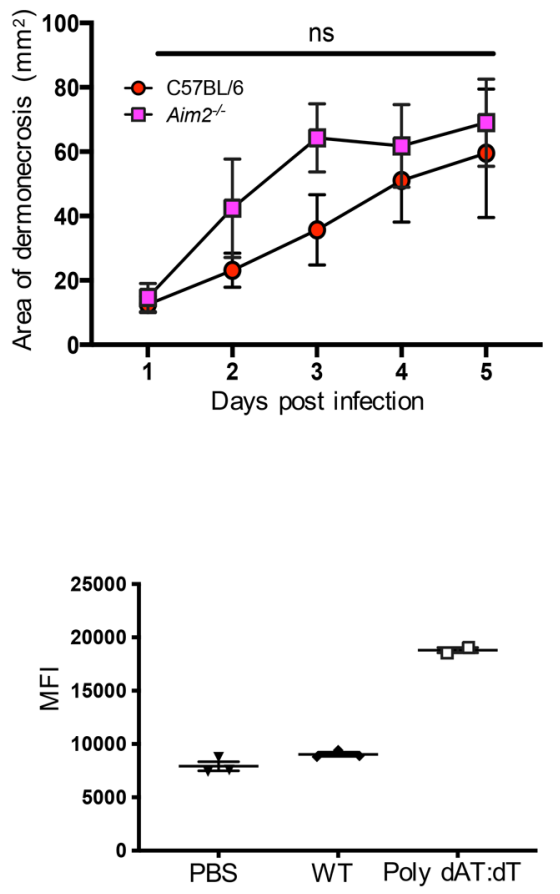

Extended data 4 | Protection against secondary S. aureus infection is independent of IL1R1-Aim2 activation. (A-B) (A) Dermonecrosis and (B) bacterial load in WT C57BL/6 and $111 \mathrm{r}^{-/-}$mice treated with PBS or infected with WT LAC on day 0 and challenged at the same site on day 28 with WT LAC; black asterisks denote statistical differences in lesion size with respect to uninfected mice, purple asterisks show statistical difference between WT C57BL/6 and $/ 11 r^{-/-}$-infected mice and green asterisks show statistical differences between $\mathrm{WT}$ C57BL/6 and $/ 11 \mathrm{r}^{-/-}$-naïve mice upon secondary challenge, $\mathrm{n}=$

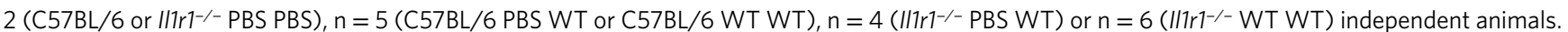
(C-D) (C) Dermonecrosis and (D) bacterial load in the skin of WT C57BL/6 and Aim2 $2^{-1-}$ mice 5 days post infection with WT LAC, ns: not significant, $n=3$ or 4 independent animals (day 1 ) or $n=10$ independent animals (day 5). (E) Intracellular mean fluorescence intensity (MFI) of Aim2 measured by flow cytometry in keratinocytes infected with WT LAC or transfected with poly dA:dT for $24 \mathrm{~h}, \mathrm{n}=1$ representative experiment from 3 independent experiments. (F) Expression of Aim 2 in HEKn cells infected with WT LAC or transfected with poly dA:dT for 24 h, compared to uninfected cells (PBS) by qRT-PCR, $n=1$ representative experiment from 3 independent experiments, For $(\mathbf{A}-\mathbf{F})$, data represent mean \pm SEM. Statistical analysis was performed by one-way (B) or two-way ANOVA (A, C and D), ${ }^{\star \star \star \star} p<0.0001,{ }^{\star \star \star} p<0.001,{ }^{\star \star} p<0.01,{ }^{\star} p<0.05, n s ; p=0.9999$ (C, day 1), 0.9051 (C, day 2), 0.6533 (C, day 3), 0.9820 (C, day 4), 0.9899 (C, day 5) and $p=0.0892$ (D, day 1) and 0.4037 (D, day 5). 


\section{Reporting Summary}

Nature Research wishes to improve the reproducibility of the work that we publish. This form provides structure for consistency and transparency in reporting. For further information on Nature Research policies, see Authors \& Referees and the Editorial Policy Checklist.

\section{Statistics}

For all statistical analyses, confirm that the following items are present in the figure legend, table legend, main text, or Methods section.

$\mathrm{n} / \mathrm{a}$ | Confirmed

$\square$ \.he exact sample size $(n)$ for each experimental group/condition, given as a discrete number and unit of measurement

$\square$ \ A statement on whether measurements were taken from distinct samples or whether the same sample was measured repeatedly

The statistical test(s) used AND whether they are one- or two-sided

$\square$ Only common tests should be described solely by name; describe more complex techniques in the Methods section.

$\bigotimes \square$ A description of all covariates tested

Х $\square$ A description of any assumptions or corrections, such as tests of normality and adjustment for multiple comparisons

A full description of the statistical parameters including central tendency (e.g. means) or other basic estimates (e.g. regression coefficient)

AND variation (e.g. standard deviation) or associated estimates of uncertainty (e.g. confidence intervals)

$\square$ For null hypothesis testing, the test statistic (e.g. $F, t, r$ ) with confidence intervals, effect sizes, degrees of freedom and $P$ value noted

$\square$ Give P values as exact values whenever suitable.

Х $\square$ For Bayesian analysis, information on the choice of priors and Markov chain Monte Carlo settings

Х $\square$ For hierarchical and complex designs, identification of the appropriate level for tests and full reporting of outcomes

$\bigotimes \square$ Estimates of effect sizes (e.g. Cohen's $d$, Pearson's $r$ ), indicating how they were calculated

Our web collection on statistics for biologists contains articles on many of the points above.

\section{Software and code}

Policy information about availability of computer code

Data collection No software used.

Data analysis We used FlowJo version 10 and GraphPad Prism version 7 to analyse the data in this study.

For manuscripts utilizing custom algorithms or software that are central to the research but not yet described in published literature, software must be made available to editors/reviewers. We strongly encourage code deposition in a community repository (e.g. GitHub). See the Nature Research guidelines for submitting code \& software for further information.

\section{Data}

Policy information about availability of data

All manuscripts must include a data availability statement. This statement should provide the following information, where applicable:

- Accession codes, unique identifiers, or web links for publicly available datasets

- A list of figures that have associated raw data

- A description of any restrictions on data availability

Data that support the findings of this study are included in this manuscript along with the supplementary figures and are also available from the corresponding author upon request. Whole genome sequence data of the $\triangle$ hemB, $\triangle$ fumC and $\triangle$ hemB $\Delta$ fumC LAC mutants and the complemented strains $\triangle$ hemB (hemB) and $\triangle$ fumC (fumC) were submitted to European Nucleotide Archive (ENA) under BioProject number PRJEB30093. 


\section{Field-specific reporting}

Please select the one below that is the best fit for your research. If you are not sure, read the appropriate sections before making your selection. \ Life sciences

$\square$ Behavioural \& social sciences Ecological, evolutionary \& environmental sciences

For a reference copy of the document with all sections, see nature.com/documents/nr-reporting-summary-flat.pdf

\section{Life sciences study design}

All studies must disclose on these points even when the disclosure is negative.

Sample size Sample sizes were large enough to detect the effects of interest holding biological significance.

Data exclusions No data were excluded.

Replication The experimental findings were reliably reproduced. Please see details in the figure legends.

Randomization Samples and animals were randomly allocated into experimental groups.

Blinding No blinding was performed.

\section{Reporting for specific materials, systems and methods}

We require information from authors about some types of materials, experimental systems and methods used in many studies. Here, indicate whether each material, system or method listed is relevant to your study. If you are not sure if a list item applies to your research, read the appropriate section before selecting a response.

Materials \& experimental systems

n/a Involved in the study

$\square$ Antibodies

$\square \bigotimes$ Eukaryotic cell lines

$\bigotimes \square$ Palaeontology

$\square$ Animals and other organisms

$\bigotimes \square$ Human research participants

\) $\square$ Clinical data

\section{Antibodies}

\author{
Methods \\ $\mathrm{n} / \mathrm{a}$ Involved in the study \\ \ $\square$ ChIP-seq \\ $\bigotimes$ Flow cytometry \\ \ $\square$ MRI-based neuroimaging
}

Antibodies used

Rabbit anti-phospho-MLKL Cell Signaling Technology Cat\# 91689

Rabbit anti-MLKL Cell Signaling Technology Cat\# 14993

Rabbit anti-phospho-S6 ribosomal protein Cell Signaling Technology Cat\# 4858

Rabbit anti-LC3B Sigma-Aldrich Cat\# L7543

Rabbit anti-TLR2 EMD Millipore Cat\# 06-1119

Rabbit anti-TNFR1 Cell Signaling Technology Cat\# 3736

Mouse anti- $\beta$-actin Sigma-Aldrich Cat\# A5316

Goat anti-rabbit-HRP Abcam Cat\# ab205718

Mouse IgGK light chain binding protein-HRP Santa Cruz Cat\# sc-516102

Brilliant Violet 605 hamster anti-mouse CD11c Clone N418 BioLegend Cat\# 117333

Brilliant Violet 421 rat anti-mouse CD86 Clone GL-1 BioLegend Cat\# 105031

Brilliant Violet 510 hamster anti-mouse CD103 Clone 2E7 BioLegend Cat\# 121423

PE-CF594 rat anti-mouse Ly6C Clone AL-21 BD Horizon Cat\# 562728

Alexa Fluor 700 rat anti-mouse CD45 Clone 30-F11 BioLegend Cat\# 103128

APC/Cy7 rat anti-mouse I-A/I-E (MHCII) Clone M5/114.15.2) BioLegend Cat\# 107628

PE mouse anti-mouse/human CD207 (Langerin) Clone 4C7 BioLegend Cat\# 144204

Alexa Fluor 594 rat anti-mouse/human CD11b Clone M1/70 BioLegend Cat\# 101254

Brilliant Violet 650 mouse anti-mouse NK-1.1 Clone PK136 BioLegend Cat\# 108735

PerCP/Cy5.5 rat anti-mouse Ly6G Clone 1A8 BioLegend Cat\# 127616

Rat anti-mouse CD16/32 (Fc block) Clone 93 BioLegend Cat\# 101302

Rabbit anti-Aim2 Abcam Cat\# ab180665

Rabbit anti-LukA, anti-LukE, anti-LukD (gift from Dr. Victor J. Torres, NYU)

Rabbit anti-Hla Sigma-Aldrich Cat\# S7531 
Policy information about cell lines

Cell line source(s)

Human epidermal keratinocytes (HEKn) Gibco Cat\# C0015C

Human monocytes (THP-1) ATCC Cat\# TIB-202

Authentication

All cell lines used were authenticated by the manufacturers and checked morphologically under the microscope.

Mycoplasma contamination

All cell lines used were routinely tested for mycoplasma contamination and were mycoplasma free.

Commonly misidentified lines

(See ICLAC register)

No commonly misidentified cell lines were used.

\section{Animals and other organisms}

Policy information about studies involving animals; ARRIVE guidelines recommended for reporting animal research

Laboratory animals

C57BL/6 Jackson Laboratory Stock No. 000664

II1r1-/- Jackson Laboratory Stock No. 003245

Aim2-/- Jackson Laboratory Stock No. 029472

Rag2 KO Jackson Laboratory Stock No. 008449

Mlkl-/- from John Silke, WEHI Australia, via Douglas R. Green, St Jude Children's Research Hospital (Murphy et al., 2013, Kitur et

al., 2016)

Ripk1d (Ripk1K45A) from Scott B. Berger, Peter J. Gough, GlaxoSmithKline (Berger et al., 2014)

Wild animals

This study did not involve wild animals.

Field-collected samples

This study did not involve samples collected from the field.

Ethics oversight

Animal work protocol (AAAR5412) was approved by the Institutional Animal Care and Use Committee (IACUC). Animal experiments were carried out in strict accordance with the recommendations in the Guide for the Care and Use of Laboratory

Animals of the $\mathrm{NIH}$, the Animal Welfare Act, and US federal law.

Note that full information on the approval of the study protocol must also be provided in the manuscript.

\section{Flow Cytometry}

Plots

Confirm that:

$\square$ The axis labels state the marker and fluorochrome used (e.g. CD4-FITC).

$\square$ The axis scales are clearly visible. Include numbers along axes only for bottom left plot of group (a 'group' is an analysis of identical markers).

All plots are contour plots with outliers or pseudocolor plots.

$\bigotimes$ A numerical value for number of cells or percentage (with statistics) is provided.

\section{Methodology}

Sample preparation

Please see the Methods section.

Instrument

BD LSR II (BD Biosciences) and BD FACS Cantoll (BD Biosciences)

Software

FlowJo version 10

Cell population abundance

No flow activated cell sorting was performed in this study.

Gating strategy

Please see the Methods section.

Tick this box to confirm that a figure exemplifying the gating strategy is provided in the Supplementary Information. 